\title{
VARIACIONES ESPACIALES Y TEMPORALES DEL FITOPLANCTON EN UN LAGO SUBTROPICAL DE ARGENTINA
}

\author{
YOLANDA ZALOCAR DE DOMITROVIC, VIVIANA M. ASSELBORN y \\ SYLVINA L. CASCO \\ Centro de Ecología Aplicada del Litoral (CECOAL, CONICET), \\ Casilla de Correo 291 (3400) Corrientes, Argentina. \\ Correspondencia para: Yolanda Zalocar de Domitrovic, Centro de Ecología Aplicada del Litoral (CECOAL, \\ CONICET), Casilla de Correo 291 (3400) Corrientes, Argentina \\ Recebido en 21/01/97 - Acceptado en 27/05/98 - Distribuido en 28/08/98
}

(Con 15 figuras)

\section{ABSTRACT \\ Temporal and spatial variations of the phytoplankton in a subtropical lake of Argentina}

Species composition, biomass and diversity of the phytoplankton in a subtropical lake $\left(27^{\circ} 29^{\prime} \mathrm{S}\right.$; $58^{\circ} 45^{\prime} \mathrm{W}$ ) were studied from February 1995 to March 1996. One or two samples were taken monthly in the limnetic and the littoral area. Cell counts were done by the Utermöhl method (1958). Phytoplankton biomass varied among 2.38 and $45.59 \mathrm{~mm}^{3} \cdot \mathrm{m}^{-2}$ with a mean value of $12.11( \pm 10.52) \mathrm{mm}^{3} \cdot \mathrm{m}^{-2}$. The population density oscillated among $0.3 \times 10^{6}$ and $4.7 \times 10^{6}$ ind. $\mathrm{cm}^{-2}$.

Cyanophyta was the dominant group all the year contributing among $25 \%$ and $83 \%$ of the total biomass. Cylindrospermopsis raciborskii, Aphanizomenon sp. and Microcystis aeruginosa were the dominant species. The Chlorophyta, second group in importance, contributed among $8 \%$ and $50 \%$ being Botryococcus braunii the dominant species. Bacillariophyceae, Xanthophyceae, Euglenophyceae and Cryptophyceae they did not surpass $20 \%$ of total biomass. Chrysophyceae and Dinophyceae they did not surpass $5 \%$ of total biomass. Chlorophyll $a$ content ranged between 0.7 and $8 \mu$ g. $\mathrm{L}^{-1}$ reflecting the annual variation of phytoplankton biomass. Phytoplankton density diversity varied among 0.34

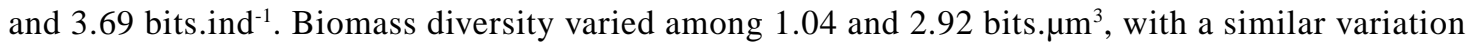
in the depths of the lake.

The lowest biomass and the highest diversity were always found in the littoral zone. C-Strategists, with a high SA/V ratios prevailed in density (Monoraphidium contortum, M. tortile, Chlorella vulgaris and other small flagellates of Chlorophyta) while S-Strategists (with a low SA/V ratios) such as Microcystis aeruginosa and Botryococcus braunii prevailed during the greater part of the year. Cylindrospermopsis raciborskii and Aphanizomenon sp. were dominant in density and biomass when the temperatures were superior to $20^{\circ} \mathrm{C}$ and the solar radiation surpassed $20 \mathrm{MJ} . \mathrm{m}^{-2}$. The R-Strategists (Aulacoseira italica and Gymnodinium sp.) prevailed during great unstability of water column.

Phytoplankton biomass was positively correlated with rain falls 10 days before each sampling $(\mathrm{r}=$ $0.772 ;$ p $<0.01 ;$ g. $1 .=30)$. During the period that prevailed the filamentous Cyanophyceae an increase in the heterocysts frequency was observed in coincidence with a period of notorous drought.

Key words: subtropical lake, phytoplankton, composition, biomass, diversity.

\section{RESUMO}

\section{Variações espaciais e temporais do fitoplâncton num lago subtropical da Argentina}

Foi estudada a composição, biomassa e diversidade das espécies de fitoplâncton num lago subtropical ( $27^{\circ} 29^{\prime}$ S; 5845'O) desde fevereiro de 1995 até março de 1996 . Uma ou duas coletas mensais fo- 
ram feitas em dois locais: uma na área central e outra na região litoral do lago. As contagens foram realizadas segundo o método de Utermöhl (1958). A biomassa do fitoplâncton variou entre 2,38 e $45,59 \mathrm{~mm}^{3} \cdot \mathrm{m}^{-2}$, com valor médio de $12,11 \mathrm{~mm}^{3} \cdot \mathrm{m}^{-2}\left( \pm 10,52 \mathrm{~mm}^{3} \cdot \mathrm{m}^{-2}\right)$. A densidade da população oscilou entre $0,3 \times 10^{6}$ e $4,7 \times 10^{6}$ ind.cm ${ }^{-2}$.

Cyanophyta predominou ao longo de todo o ano, contribuindo com entre $25 \%$ e $83 \%$ para a biomassa total. Cylindrospermopsis raciborskii, Aphanizomenon sp. e Microcystis aeruginosa foram as espécies melhor representadas . Chlorophyla, segundo grupo em importância, apresentou-se entre 8\% a 50\%, sendo Botryococcus braunii a espécie dominante. Bacillariophyceae, Xanthophyceae, Euglenophyceae e Cryptophyceae não superaram 20\% da biomassa total. O aporte de Chrysophyceae e Dinophyceae à biomasa total não ultrapassou $5 \%$. O conteúdo de clorofila $a$ variou entre 0,7 e $8 \mu \mathrm{g} . \mathrm{L}^{-1}$, refletindo a variação anual da biomassa do fitoplâncton. A diversidade baseada na densidade do fitoplâncton variou entre 0,34 e 3,69 bits.ind ${ }^{-1}$ e a diversidade baseada na biomassa oscilou entre 1,04 e 2,92 bits.ind ${ }^{-1}$, com variação similar nas diferentes profundidades do lago.

A mais baixa biomassa e a mais alta diversidade foram sempre registradas na região litoral. Os estrategistas $\mathrm{C}$, com alta relação AS/V, dominaram em densidade (Monoraphidium contortum, M. tortile, Chlorella vulgaris e outros pequenos flagelados de Chlorophyta), enquanto os estrategistas $\mathrm{S}$, com baixa relação AS/V, tais como Microcystis aeruginosa e Botryococcus braunii, predominaram em biovolume durante a maior parte do ano. Cylindrospermopsis raciborskii e Aphanizomenon sp. dominaram em densidade e biomassa quando a temperatura foi superior a $20^{\circ} \mathrm{C}$ e a radiação solar, a $20 \mathrm{MJ} . \mathrm{m}^{-2}$. Os estrategistas R (Aulacoseira italica e Gymnodinium sp.) dominaram quando a coluna d'água apresentou grande instabilidade.

A biomassa do fitoplâncton correlacionou-se positivamente com a precipitação ocorrida dez dias antes de cada amostragem $(\mathrm{r}=0,772 ; \mathrm{p}<0,01$; g.l. = 30). Durante o tempo em que predominaram as Cyanophyceae filamentosas, observou- se um aumento na freqüência de heterocistos, coincidentemente em épocas de notória estiagem.

Palavras-chave: lago subtropical, fitoplâncton, composição, biomassa, diversidade.

\section{INTRODUCCION}

Los estudios ecológicos del fitoplancton en ambientes tropicales son escasos y recientes (Lewis, 1978a,b, 1986; Reynolds, 1984; Talling, 1986; Huszar, 1989; Marinho et al.,1990, 1993) en comparación con los producidos para lagos templados del hemisferio norte. La región subtropical de Argentina no constituye una excepción, donde la mayoría de los trabajos fueron realizados en ambientes lénticos del valle de inundación del río Paraná (García de Emiliani, 1980, 1990, 1993; Zalocar de Domitrovic, 1990, 1992, 1993).

La "Laguna Aeroclub" (en el NO de la provincia de Corrientes) forma parte de un conjunto de ambientes que ocupan aproximadamente un $30 \%$ de la superficie de la cuenca del Riachuelo, un afluente del río Paraná. Estudios de fitoplancton en estos ambientes sólo comprenden muestreos estacionales u ocasionales incluidos en investigaciones limnológicas (Bonetto et al., 1978a, 1978b), de su producción primaria (Caro et al., 1979) y de la interacción fito-zooplancton (Matveev et al., 1992).

Este trabajo comprende un estudio de las variaciones espaciales y temporales del fitoplancton en un lago subtropical, analizando su composición taxonómica, biomasa y diversidad específica en relación a las principales variables ambientales.

\section{AREA DE ESTUDIO}

El lago (Fig. 1), denominado "Laguna Aeroclub" ( $\left.27^{\circ} 29^{\prime} S ; 58^{\circ} 45^{\prime} \mathrm{O}\right)$, posee forma redondeada, una superficie de aproximadamente $26 \mathrm{Ha}$ y una profundidad media de 3,5 metros (Zmáx = $6 \mathrm{~m}$.). Su régimen hídrico depende directamente de las precipitaciones, cuyas medias anuales oscilan entre 1100 y $1200 \mathrm{~mm}$. Presenta las ca- 
racterísticas de un lago polimíctico cálido, según la clasificación de Hutchinson (1957), eutrófico y con limitación de nutrientes, principalmente de fósforo (Zalocar de Domitrovic et al., en prep.). El clima de la región es subtropical de transición, con predominante influencia marítima (Bruniard, 1981).

Durante los estudios, la vegetación de la zona litoral estuvo representada por Cyperus sp., Polygonum sp., Salvinia herzogii De la Sota, Azolla caroliniana Willd. e Hydrocleis nymphoides (Willd.) Buchenau. El fondo de la laguna y hasta aproximadamente los 3,5 metros de profundidad estuvo vegetado por Egeria sp. y Nitella sp.

Algunos sectores de arena, de color verde azulado, estuvieron colonizados por Cyanophyceae psammíticas (Chlorogloea microcystoides Geit., Plectonema gracillimum (Zopf) Hansg. y Myxosarcina spectabilis Geit.) formando un estrato de unos $10 \mathrm{~cm}$ de espesor.

\section{MATERIAL Y METODOS}

Se establecieron dos puntos de muestreo: uno en la zona central y más profunda del lago (Estación A) y otro en el área litoral, próximo a la zona vegetada (Estación B). La frecuencia de los muestreos fue generalmente de periodicidad mensual y ocasionalmente quincenal, durante 14 meses (desde febrero de 1995 hasta marzo de 1996). En la zona litoral, los muestreos se iniciaron en abril/95.

Para cuantificar las poblaciones de algas, las muestras se extrajeron con botella Van Dorn de $2 \mathrm{~L}$ de capacidad, y fueron fijadas in situ con lugol acético. En la zona limnética se recolectaron a tres profundidades: una en la zona subsuperficial, otra a una profundidad media de la profundidad total de la laguna y la última en las proximidades del fondo. En la costa, en cambio, dada la escasa profundidad (aproximadamente 1,30 m.), las muestras fueron extraídas sólo en el estrato superfi-

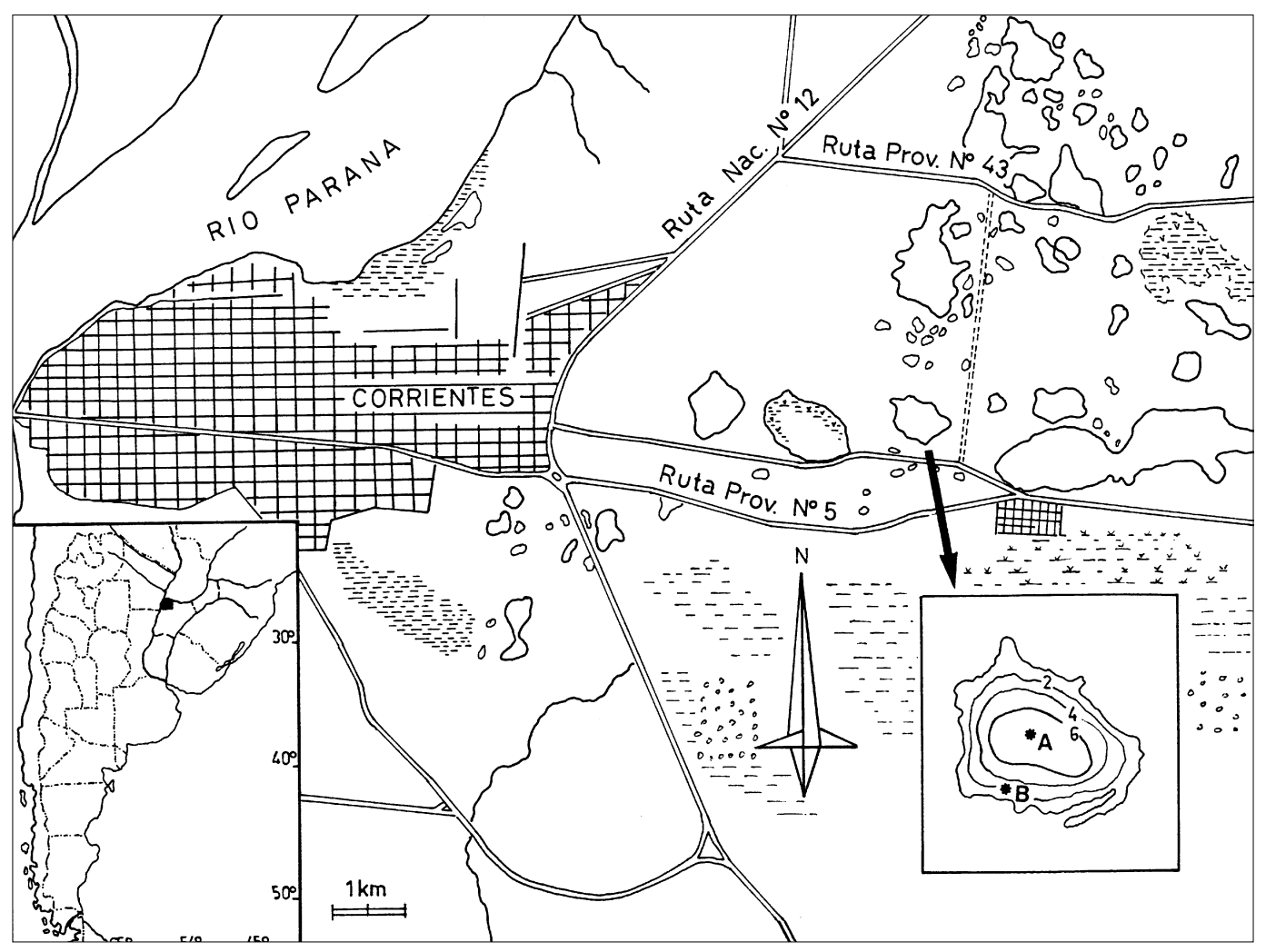

Fig. 1 - Ubicación de la "laguna Aeroclub" en el NO de la provincia de Corrientes (Argentina) y estaciones de muestreo (A: zona limnética, B: zona litoral). 
cial. La estimación de la biomasa fue realizada por el método de Utermöhl (1958). Fueron contados como mínimo 100 individuos (células, cenobios, colonias y/o filamentos) de la especie más frecuente, de modo que el error de recuento fuese inferior al 20\%, con un nivel de significación del 95\% (Lund et al., 1958). Las algas más grandes fueron contadas con menor aumento $(63 \mathrm{x})$ recorriendo la totalidad del fondo de la cámara sedimentada $\left(10 \mathrm{~cm}^{3}\right)$.

El volumen celular $\left(\mu \mathrm{m}^{3}\right)$ fue calculado para todas las especies sobre la base de mediciones de 30-100 individuos (entre 5 y 10 para los taxones más raros). Las especies fueron aproximadas a formas geométricas simples o combinadas (Rott, 1981) para estimar el volumen celular $\left(\mathrm{mm}^{3} \mathrm{~L}^{-1}=\right.$ $\mathrm{mg}$ de peso fresco $\mathrm{L}^{-1}$ ). Algunos cálculos fueron simplificados mediante el empleo de las tablas de volumen de Senichkin (1978).

La diversidad específica (H') fue calculada de acuerdo a Shannon \& Weaver (1963) y la equidad (E\%) como 100. H'/H' máx.

Se realizaron mediciones de algunas variables físicas y químicas del agua, en la zona limnética, tales como: transparencia (con disco de Secchi), temperatura (con teletermómetro adosado al conductímetro y oxímetro), conductividad eléctrica (con conductímetro YSI 33 SCT), pH (con peachímetro digital Metrohm A G Herisau) y oxígeno disuelto (con oxímetro YSI $54 \mathrm{~A}$ ).

La clorofila $a$ fue extraída con acetona $90 \%$ estimando su concentración según las ecuaciones de APHA (1985). Los análisis de los nutrientes disueltos: nitrógeno (amonio, nitritos + nitratos), fósforo (ortofosfatos), fueron realizados de acuerdo a las instrucciones señaladas en APHA (1985).

Las variables abióticas y bióticas fueron correlacionadas utilizando el coeficiente de correlación de Pearson. Para comparar estadísticamente las diferentes profundidades y estaciones de muestreo se utilizó ANOVA y las pruebas F de Snedecor y $t$ de Student (Sokal y Rohlf, 1979). Los datos fueron normalizados mediante transformación $\log (\mathrm{n}+1)$.

\section{RESULTADOS}

\section{Variables ambientales}

La media mensual de temperatura del aire varió entre $18^{\circ} \mathrm{C}$ (agosto/95) y $26^{\circ} \mathrm{C}$ (diciembre/
95). Las temperaturas máximas se registraron durante los meses de diciembre y enero $\left(41^{\circ} \mathrm{C}\right)$ y las mínimas en julio y agosto $\left(1^{\circ} \mathrm{C}\right)$.

La media mensual de radiación solar (Fig. 4) varió entre $10 \mathrm{MJ} \cdot \mathrm{m}^{-2}$ (en junio y agosto/95) y $27 \mathrm{MJ} . \mathrm{m}^{-2}$ (en noviembre/95 y enero/96).

El año 1995 se caracterizó por sus escasas precipitaciones (Fig. 2). Estas estuvieron distribuidas en forma heterogénea a lo largo del año, pero la mayor pluviosidad se dio a fines de verano y durante el otoño de 1995. La velocidad media mensual del viento (Fig. 2) varió entre $0,8 \mathrm{~m} \cdot \mathrm{seg}^{-1}$ (mayo/95) y $2,3 \mathrm{~m} \cdot \mathrm{seg}^{-1}$ (noviembre/95).

La Fig. 3 representa las principales variables ambientales medidas in situ. Las lecturas del disco de Secchi oscilaron entre 89 y $270 \mathrm{~cm}$. La temperatura del agua (entre 14,3 y $28^{\circ} \mathrm{C}$ ), presentó en general un perfil vertical bastante homogéneo con escasa variación térmica. Durante primavera y verano se registraron zonas de discontinuidad térmica a partir de los 3 metros de profundidad, con variaciones de 1,7 a $3^{\circ} \mathrm{C}$ entre la superficie y el fondo.

La conductividad eléctrica presentó un rango de variación entre 50 y $77 \mu \mathrm{S} . \mathrm{cm}^{-1}$ entre superficie y aproximadamente los 4 metros, incrementándose a partir de esta profundidad para alcanzar sus valores máximos en las proximidades del fondo (entre 60-241. $\mu$ S.cm ${ }^{-1}$ ).

$\mathrm{El} \mathrm{pH}$ (entre 5,8 y 8 unidades), de un modo general fue más elevado en el estrato superficial o a profundidades intermedias, probablemente en relación a una mayor actividad fotosintética.

El oxígeno disuelto, de la misma manera que el $\mathrm{pH}$, presentó valores decrecientes hacia el fondo con un valor medio de 8,7 en superficie; 8,3 alrededor de los $2,50 \mathrm{~m}$. y $3,5 \mathrm{mg} . \mathrm{L}^{-1}$ en las proximidades del fondo. El porcentaje de saturación varió entre 7,8\% y 9,9\%, con sobresaturación en superficie y profundidad media en la mayor parte de los muestreos.

La concentración de nitrógeno, como amonio y nitritos + nitratos, varió en un rango desde no detectable hasta 125 y $26 \mu \mathrm{g} . \mathrm{L}^{-1}$, respectivamente (Fig. 15) con severas limitaciones hacia el final de los estudios. El fósforo (como ortofosfatos) no fue detectable durante la mayor parte del período, con la única excepción del muestreo realizado el 2-11-95, en que alcanzó concentraciones de $6 \mu \mathrm{g} . \mathrm{L}^{-1}$. 


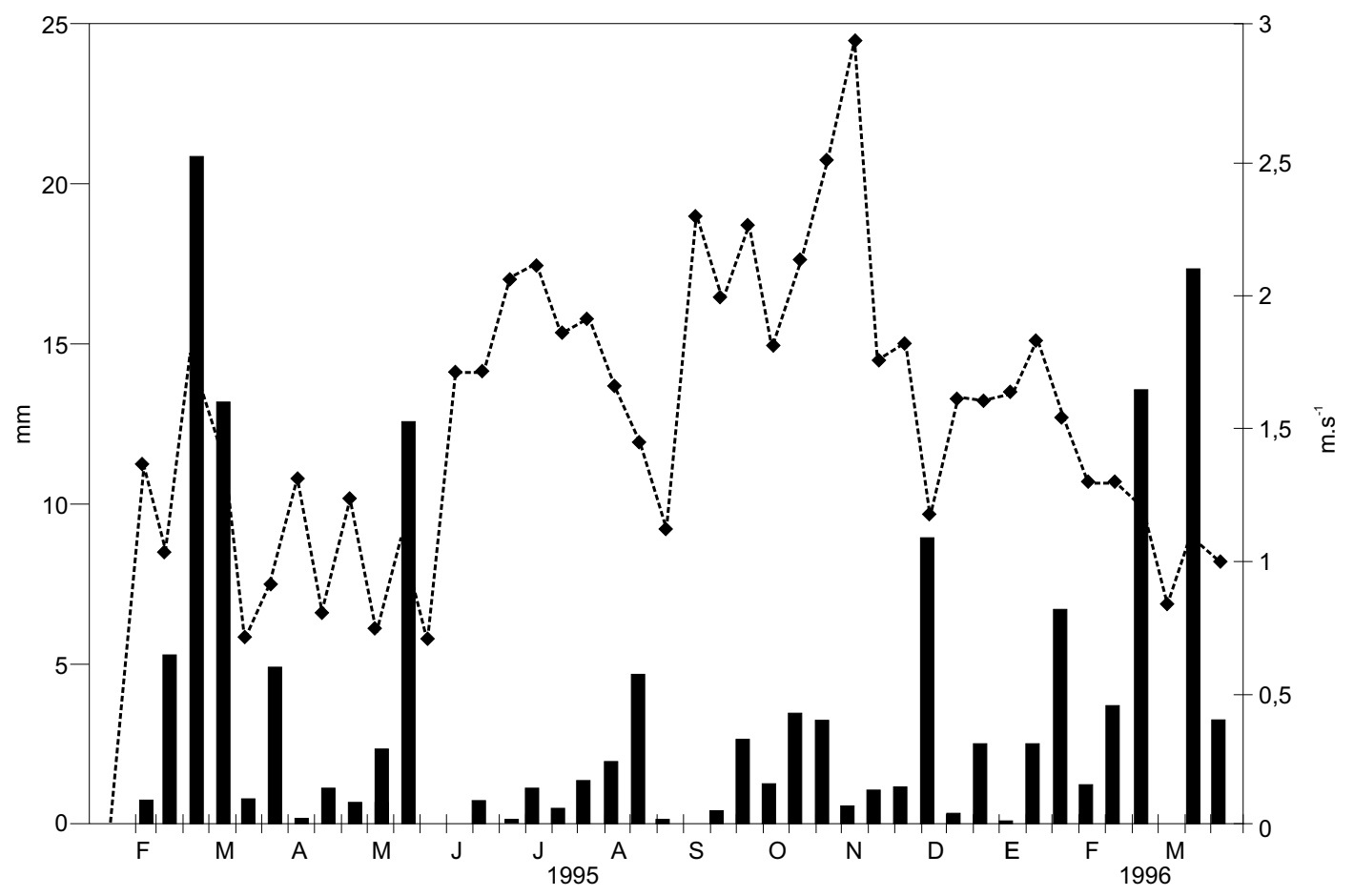

Fig. 2 - Variaciones de la pluviosidad (barras) y de la velocidad media del viento (líneas) expresados como valores medios por década, durante el período de estudios.

\section{a) Fitoplancton}

Se registraron en total 137 taxones de algas de las siguientes Clases: Cyanophyceae (21), Chlorophyceae (47), Ulothricophyceae (4), Conjugatophyceae (26), Bacillariophyceae (6), Chrysophyceae (4), Xanthophyceae (8), Euglenophyceae (11), Cryptophyceae (7) y Dinophyceae (3). De todos ellos 86 se registraron en el área limnética (Estación A), 125 en la zona litoral (Estación B) y 74 fueron comunes a ambas.

Valores extremos de biomasa se presentaron durante el verano-otoño (máximos) e invierno de 1995 (mínimos). En la zona limnética la biomasa varió entre 2,38 $\mathrm{mm}^{3} \cdot \mathrm{m}^{-2}$ (22-8-95) y 45,59 $\mathrm{mm}^{3} \cdot \mathrm{m}^{-2}$ (28-2-95), con un valor medio anual de $12,11 \mathrm{~mm}^{3} \cdot \mathrm{m}^{-2}( \pm 10,52)$. La densidad osciló entre $0,3 \times 10^{6}$ ind. $\mathrm{cm}^{-2}$ y $4,7 \times 10^{6}$ ind. $\mathrm{cm}^{-2}$. En la zona litoral, en cambio, ambas variables registraron valores inferiores al de la zona limnética (Fig. 5).

Cyanophyta fue el grupo dominante a lo largo del año, contribuyendo entre el 25 y el $83 \%$ al total de la biomasa (Fig. 6). Las Chlorophyta, segundo grupo en importancia, en cambio, contri- buyeron entre el 8 y el 50\%. Bacillariophyceae, Xanthophyceae, Euglenophyceae y Cryptophyceae presentaron valores inferiores al $20 \%$ mientras que Chrysophyceae y Dinophyceae, no superaron el 5\% de la biomasa total.

Dos fueron los grupos que dominaron en densidad a lo largo del año: Cyanophyta (entre el 19 y 83\%) y Chlorophyta (entre el 1 y $81 \%$ ). Sólo ocasionalmente dominaron las Cryptophyceae (32\%) a fines de invierno (12-9-95). Los grupos restantes no superaron el $2 \%$ de la densidad total (Fig. 7).

El número de especies dominantes en biovolumen, en la zona limnética, a diferentes profundidades, osciló entre 6 y 7 y, en densidad numérica fue un poco mayor, entre 9 taxones en superficie y profundidad media y 12 en proximidades del fondo. En la zona litoral, predominaron 4 y 6 taxones en biomasa y densidad, respectivamente.

Las dimensiones algales variaron entre 20 $\mu \mathrm{m}^{3}$ (Monoraphidium contortum) y $18,8 \times 10^{6} \mu \mathrm{m}^{3}$ (Botryococcus braunii). 


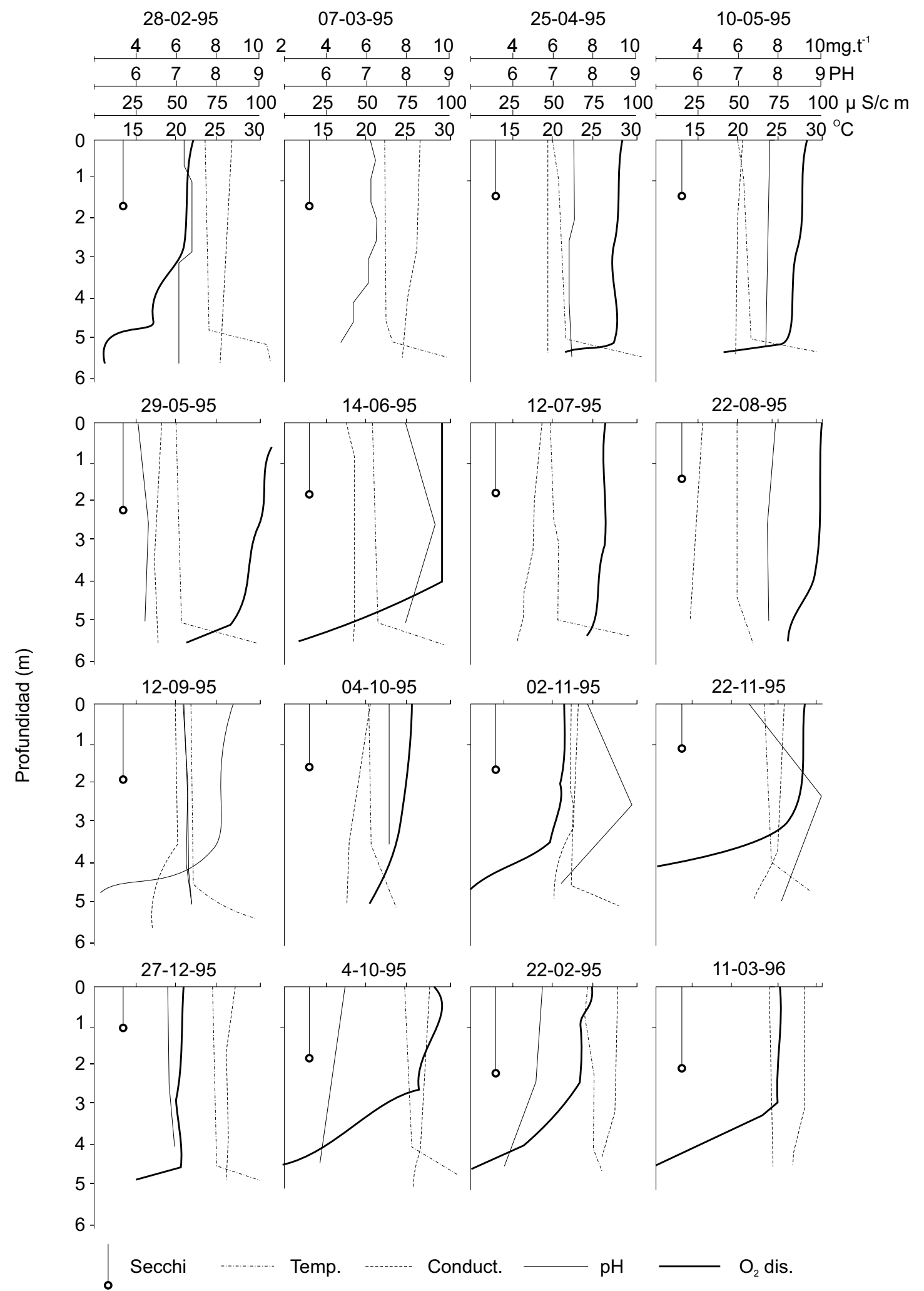

Fig. 3 - Variaciones verticales de las principales variables ambientales medidas in situ durante el período de estudios. 


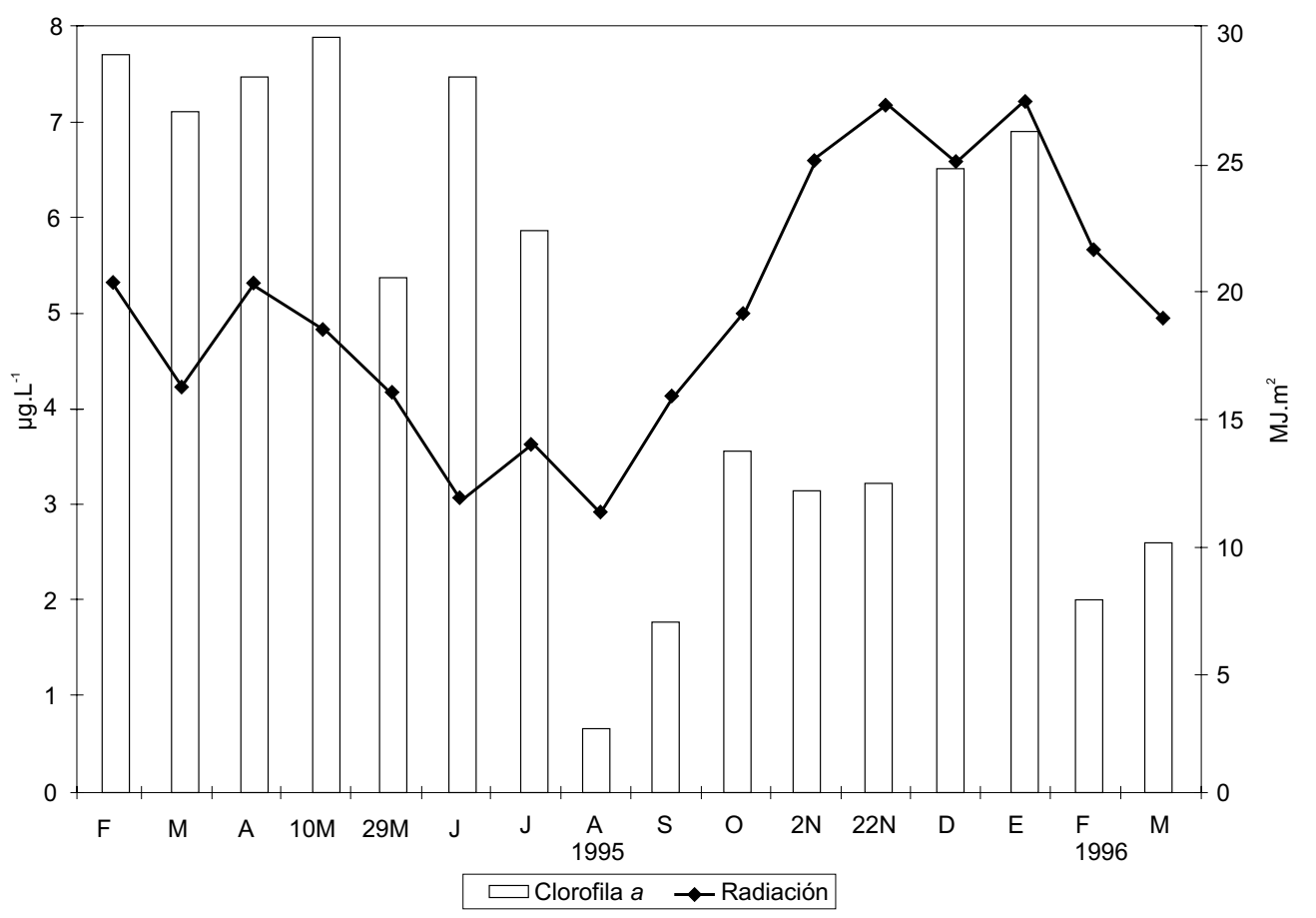

Fig. 4 - Variaciones de la radiación solar media mensual $\left(\mathrm{MJ} . \mathrm{m}^{-2}\right)$ y de la clorofila $a\left(\mu \mathrm{g} . \mathrm{L}^{-1}\right)$ en el período de estudios.

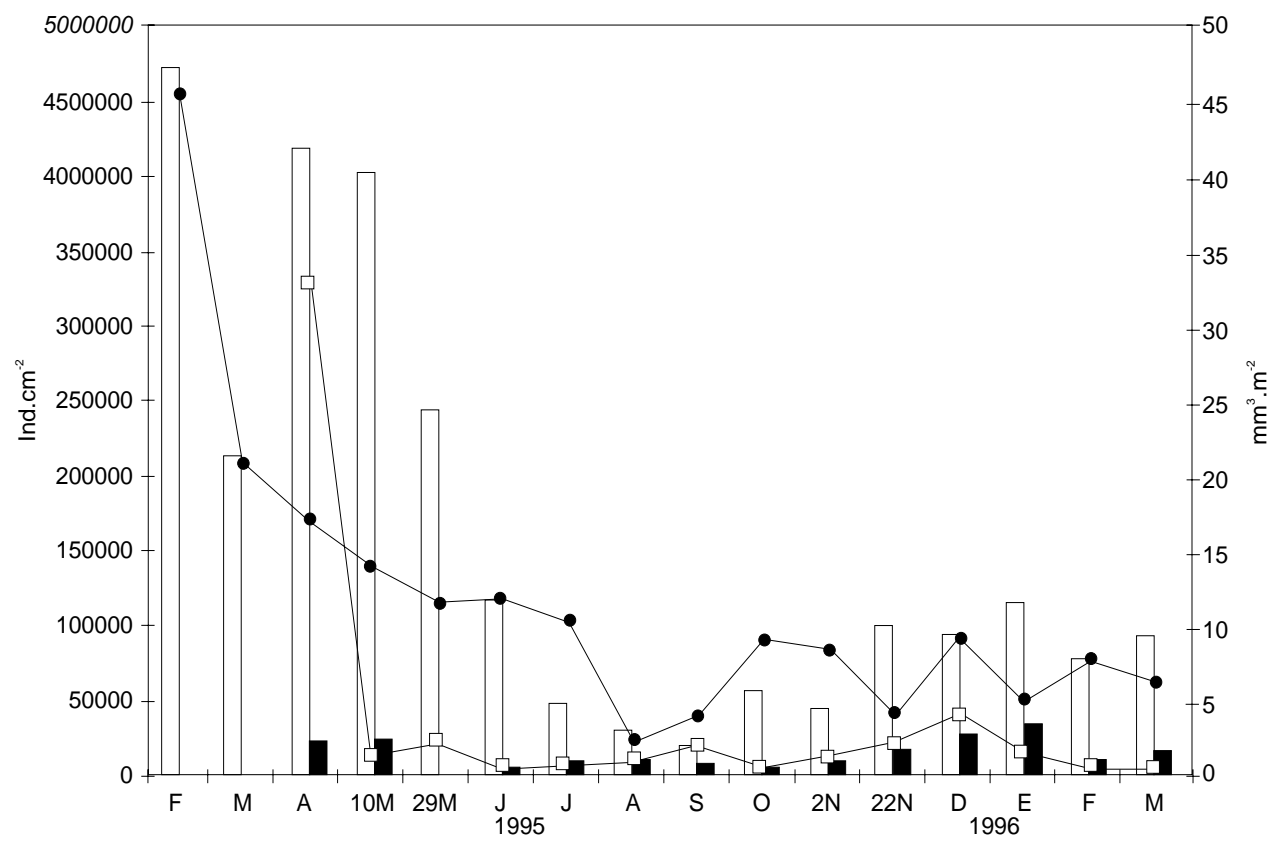

$\square$ Densidad (Z. Limnética) $\square$ Densidad (Z. Litoral) $\quad \bullet-$ Biovol. (Z. Limnética) $\quad \square-$ Biovol. (Z. Litoral)

Fig. 5 - Variaciones de la densidad (ind. $\left.\mathrm{cm}^{-2}\right)$ y del biovolumen $\left(\mathrm{mm}^{3} \cdot \mathrm{m}^{-2}\right)$ del fitoplancton en las dos estaciones de muestreo. 


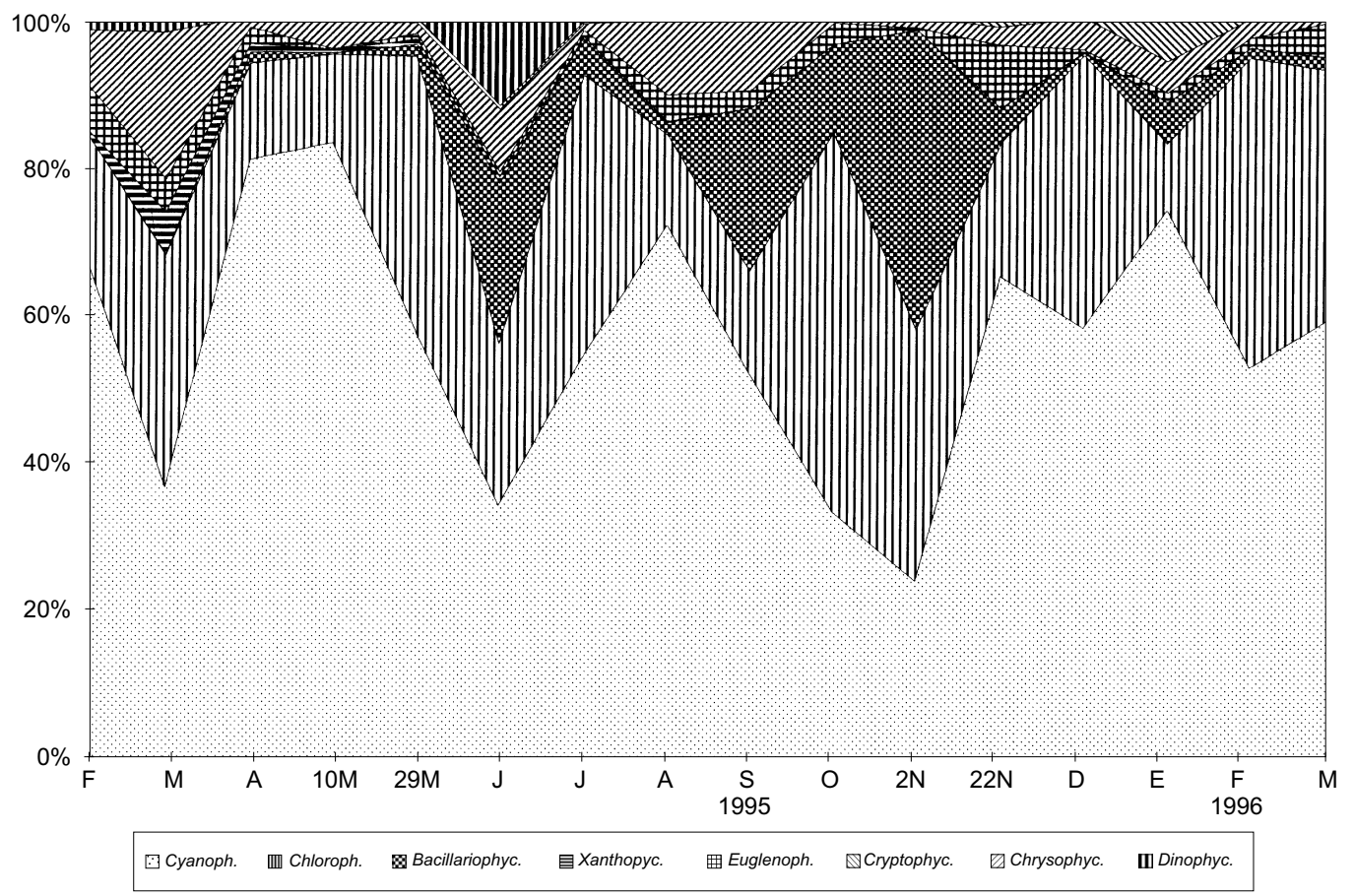

Fig. 6 - Variación temporal de la biomasa del fitoplancton en términos de volumen celular $\left(\mathrm{mm}^{3} \cdot \mathrm{m}^{-2}\right)$ expresada como porcentaje de la biomasa total.

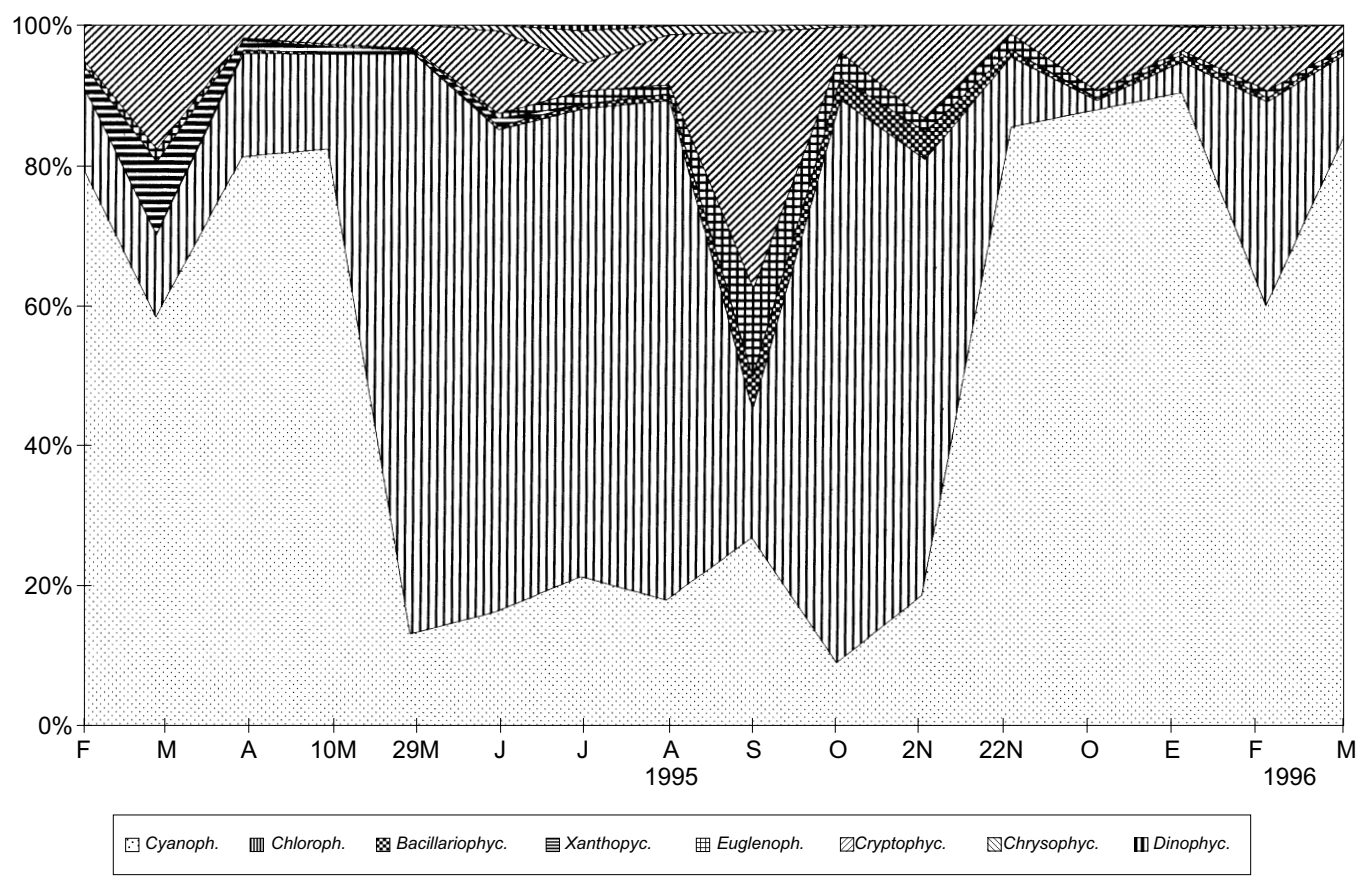

Fig. 7 - Variaciones de la densidad del fitoplancton (ind. $\mathrm{cm}^{-2}$ ) expresadas como porcentaje de la densidad total, durante el período de estudios. 
El período de estudios fue dividido en cuatro intervalos de tiempo (períodos A, B, C y D señalados en las Figs. 8-10), separados teniendo en cuenta las especies dominantes y los factores ambientales.

\section{b) Variaciones en la composición taxonómica y biomasa del fitoplancton.}

CYANOPHYTA: predominaron durante los estudios, con la excepción de los muestreos de junio y principios de noviembre de 1995, en que fueron superadas ligeramente por Bacillariophyceae y Chlorophyta (Fig. 6).

Cinco fueron las especies responsables de la elevada biomasa en este grupo: Cylindrospermopsis raciborskii, Aphanizomenon sp., Phormidium mucicola, Microcystis aeruginosa y Merismopedia tenuissima, las que contribuyeron a su totalidad con valores de hasta el $86 \%, 90 \%, 64 \%$, $63 \%$ y $33 \%$, respectivamente (Figs. 8-11).

Cylindrospermopsis raciborskii y Aphanizomenon sp. (371 y $5914 \mu \mathrm{m}^{3}$, respectivamente) fueron las especies dominantes, tanto en biomasa como en densidad, durante los meses más cálidos del año (períodos A, B y D de la Fig. 8).
Colonias de Microcystis aeruginosa $(2,5 \mathrm{x}$ $10^{6} \mu \mathrm{m}^{3}$ ) se registraron durante todo el año, pero fueron más abundantes durante el período $\mathrm{C}$ en todo el perfil vertical, manteniendo así elevados valores de biomasa inclusive durante el invierno, período en que no se detectaron otras cianofíceas. En el área limnética, si bien no se observaron diferencias estadísticamente significativas a las diferentes profundidades muestreadas, tanto el mayor número de colonias (15000 col.L $\left.\mathrm{L}^{-1}\right)$ como de su biomasa $\left(2,8 \mathrm{~mm}^{3} \cdot \mathrm{L}^{-1}\right)$ se registró generalmente en las proximidades del fondo (Fig. 10) y en la zona litoral (Fig. 11). Phormidium mucicola, asociada al mucílago de $M$. aeruginosa, sólo ocasionalmente se presentó libre en el plancton siendo dominante en densidad (95\%) y biovolumen (64\%) en el muestreo del 22-11-95, a profundidades intermedias entre la superficie y el fondo (Fig. 9). La dominancia de esta especie coincidió con el colapso de las colonias de M. aeruginosa.

CHLOROPHYTA: la mayor contribución a la biomasa total estuvo dada por Botryococcus braunii, cuyas colonias presentaron amplias variaciones de tamaño a diferentes profundidades a lo largo del año. En la zona limnética el número má-

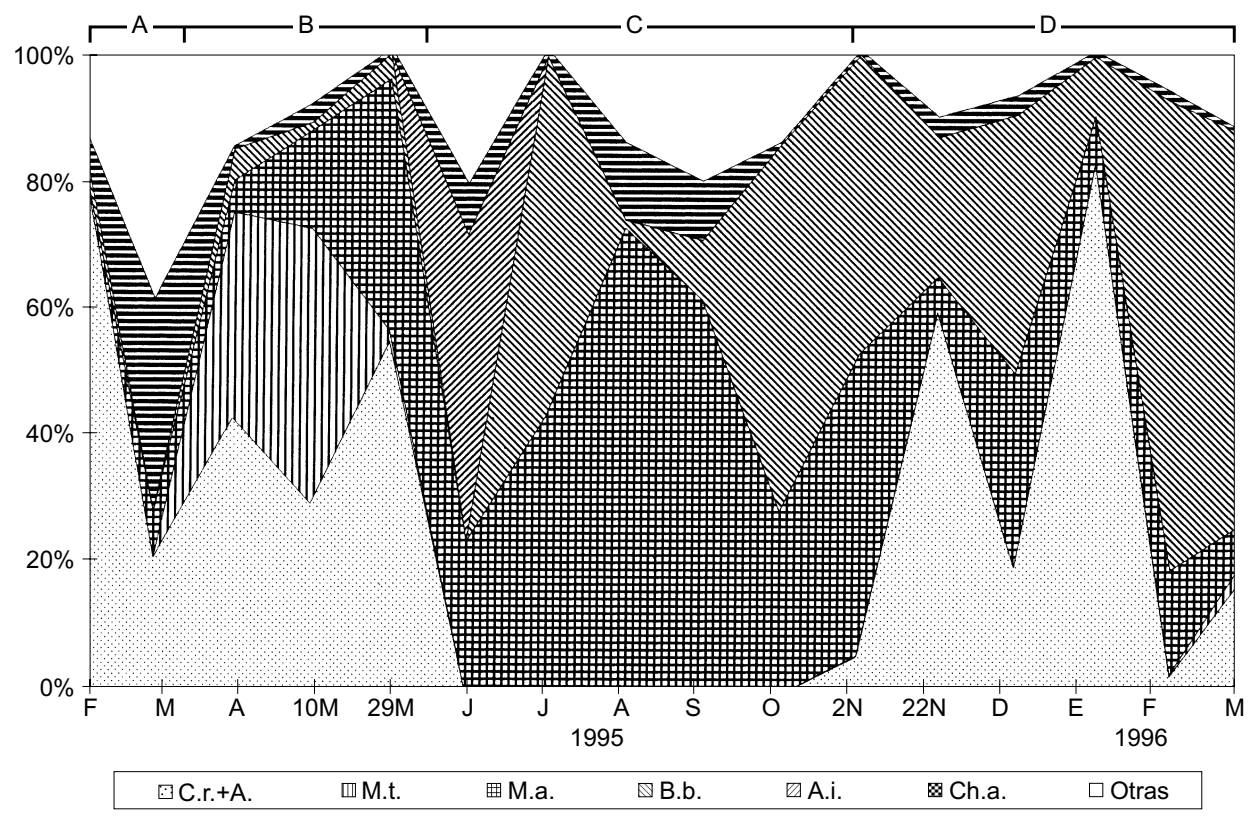

Fig. 8 - Biomasa $\left(\mathrm{mm}^{3} . \mathrm{L}^{-1}\right)$ de las especies dominantes del fitoplancton (\% de la biomasa total) en el estrato superficial de la zona limnética del lago. C.r. + A.: Cylindrospermopsis raciborskii + Aphanizomenon sp., M.t.: Merismopedia tenuissima, M.a.: Microcystis aeruginosa, B.b.: Botryococcus braunii, A.i: Aulacoseira italica, Cr. spp.: Cryptomonas erosa + Cryptomonas ovata, Otras: otras especies no dominantes. A, B, C y D: períodos en el estudio del fitoplancton. 
ximo de colonias por litro fue de 5000, $7000 \mathrm{y}$ 12000 en superficie, profundidad media y proximidades del fondo, respectivamente. A diferencia de lo señalado para $M$. aeruginosa, en $B$. braunii se dio una relación inversa entre número de colonias y volumen celular. Es así que en el estrato superficial su contribución a la biomasa total alcanzó valores del $45 \%$ (4-10-95) y $62 \%$ (22-2-96) (Fig. 8), en profundidades intermedias, del 56\% (4-10-95) y $85 \%$ (11-3-96) (Fig. 9) y, valores inferiores al 32\% (14-6-95 y 27-12-95) en las proximidades del fondo (Fig. 10). En la zona litoral, el mayor número de colonias de $B$. braunii (10000 col. $\left.\mathrm{L}^{-1}\right)$ se registró en el muestreo del 22-11-95, sin embargo, debido a su pequeño tamaño, su contribución a la masa total, no superó el 5\%. El máximo biovolumen $\left(20 \times 10^{6} \mu \mathrm{m}^{3}\right)$ se registró el 25-4-95 (con 6000 col.L $\mathrm{L}^{-1}$ ) y su contribución a la biomasa total superó ligeramente el 95\% (Fig. 11).

Al declinar las Cyanophyceae, las Chlorophyta ocuparon el primer lugar en importancia numérica (ind. $\mathrm{mL}^{-1}$ ), fundamentalmente en los estratos medio y superficial. Por lo general, no siempre se registraron las mismas especies dominantes a las diferentes profundidades. En el estrato superficial predominaron Chlorella vulgaris y Monoraphidium tortile, a profundidades intermedias, además de Monoraphidium tortile, pequeños flagelados y Sphaerocystis planctonica $\mathrm{y}$ en las proximidades del fondo, pequeños flagelados verdes, Monoraphidium tortile, $M$. contortum y clorofíceas cocoides. El aporte de estas especies a la biomasa total no superó el $2 \%$.

En la zona litoral se observó un patrón de variación similar al de la zona limnética de la laguna, pero aquí únicamente dos especies de Chlorophyta fueron dominantes en densidad numérica: Monoraphidium contortum y M. tortile.

CRYPTOPHYCEAE: de presencia constante a lo largo del año, estuvieron representadas fundamentalmente por Cryptomonas ovata $\left(888 \mathrm{~mm}^{3}\right)$ y Cryptomonas erosa $\left(559 \mu \mathrm{m}^{3}\right)$. Su contribución a la biomasa total alcanzó hasta el 34\% (el 7-395) en el estrato superficial (Fig. 8). Sólo ocasionalmente fueron dominantes numéricos en la zona litoral, con el 60\% (14-6-95) y en las proximidades de la superficie, en el área limnética, con el 34\% (el 12-9-95).

BACILLARIOPHYCEAE: fueron escasas y no superaron el $2 \%$ de la densidad total. La especie dominante fue Aulacoseira italica, de biovolumen variable según la longitud de los filamentos (células de $687 \mu \mathrm{m}^{3}$ ). Las poblaciones de esta especie, estuvieron limitadas a las proximidades del fondo (Fig.10) y a la zona litoral (Fig. 11), contribuyendo hasta el 54\% de la biomasa total. Sólo ocasionalmente y en un único muestreo (14-6-95) se presentó en el estrato superficial (Fig. 8) contribuyendo con el 50\% a la biomasa total.

XANTHOPHYCEAE: sólo se presentaron a fines de verano y durante el otoño de 1995, no superando el $5 \%$ y el $8 \%$ de densidad y biomasa total, respectivamente, en el muestreo del 7-3-95. La pequeña Xanthophyceae Chlorogibba allorgei $\left(697 \mu \mathrm{m}^{3}\right)$ contribuyó con el $74 \%$ a la biomasa total de este grupo. Pseudostaurastrum lobulatum $\left(2150 \mu^{3}\right)$, Tetraedriella jovetii $\left(540 \mu \mathrm{m}^{3}\right)$ у $T e$ traplektron torsum $\left(6468 \mu \mathrm{m}^{3}\right)$ sólo se registraron esporádicamente.

CHRYSOPHYCEAE: se observaron ocasionalmente aportando hasta el 3,3\% a la densidad (el 12-7-95) y hasta el 5\% a la biomasa total (el 4-1-96), principalmente con dos especies del género Mallomonas $\left(452 \mu \mathrm{m}^{3}\right.$ y $\left.1238 \mu \mathrm{m}^{3}\right)$.

DINOPHYCEAE: también de presencia esporádica, estuvieron representadas por Peridinium sp. $\left(4053 \mu \mathrm{m}^{3}\right)$ y Gymnodinium sp. $\left(1767 \mu \mathrm{m}^{3}\right)$, contribuyendo este último con valores de hasta el $10 \%$ a la biomasa total, a fines de otoño (14-6-95).

EUGLENOPHYCEAE: Euglena sp. (1105 $\left.\mu \mathrm{m}^{3}\right)$, Phacus sp. $\left(912 \mu \mathrm{m}^{3}\right)$ se encontraron en los estratos superficial e intermedio. Trachelomonas volvocina $\left(905 \mu \mathrm{m}^{3}\right)$ y $T$. armata $\left(2700 \mu \mathrm{m}^{3}\right)$ se observaron en todas las profundidades, pero la primera alcanzó mayor importancia numérica (17\%) en el muestreo del 4-10-95, en las proximidades del fondo.

\section{c) Variaciones de la comunidad en relación a va- riables ambientales}

Los estudios de fitoplancton fueron divididos en cuatro intervalos de tiempo (Figs. 8-10). Si bien se tuvieron en cuenta las variaciones de algunos de los factores que controlan su crecimiento (radiación solar, temperatura, nutrientes, etc.) estos intervalos fueron separados de acuerdo a cambios producidos en las especies dominantes y a períodos de circulación (situaciones en que las variables físicas y químicas fueron homogéneas entre superficie y las proximidades del fondo). 


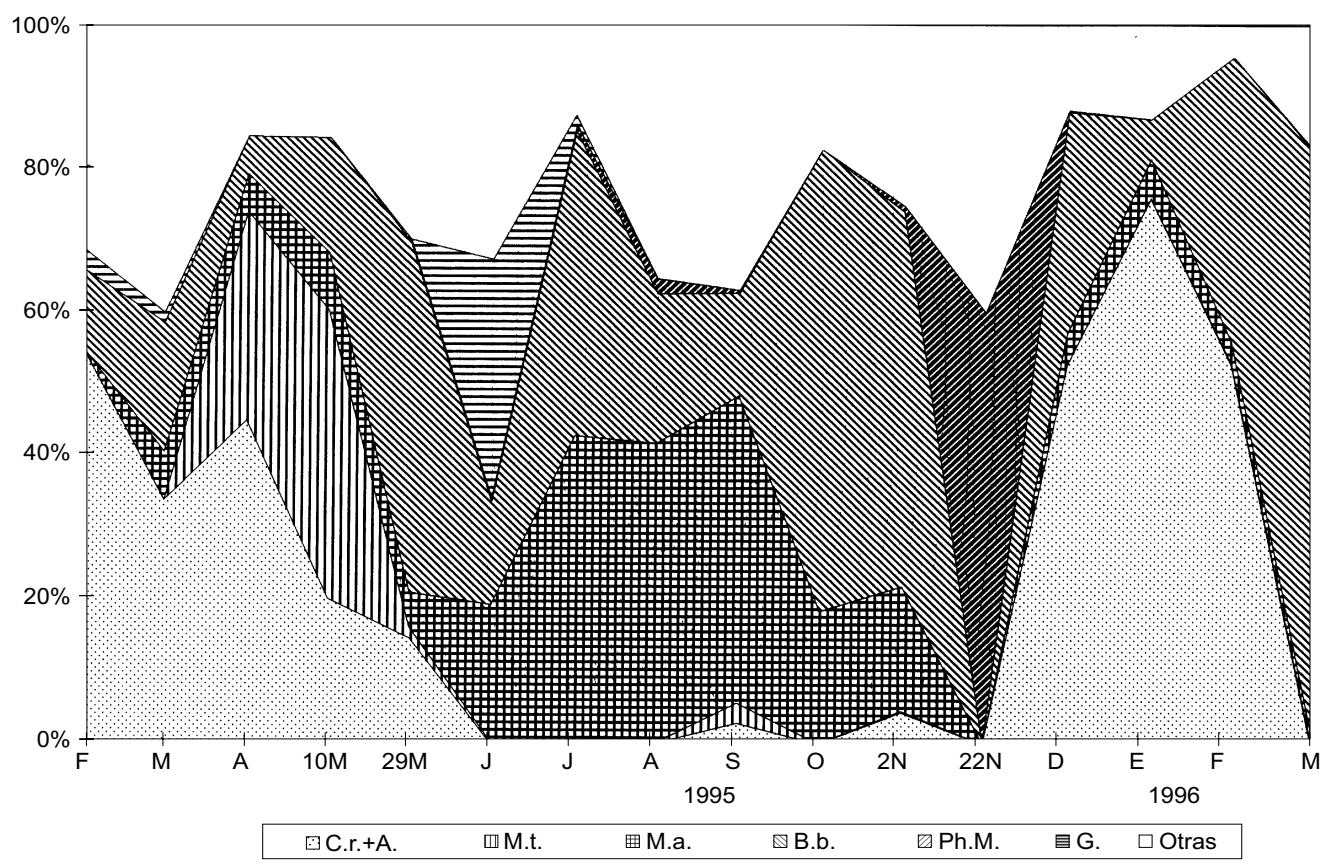

Fig. 9 - Variación temporal de la biomasa relativa de las especies dominantes del fitoplancton en el estrato intermedio (entre la superficie y el fondo) de la zona central. C.r. + A.: Cylindrospermopsis raciborskii + Aphanizomenon sp., M.t.: Merismopedia tenuissima, M.a.: Microcystis aeruginosa, B.b.: Botryococcus braunii, Ph.m.: Phormidium mucicola, G.: Gymnodinium, Otras: otras especies no dominantes.

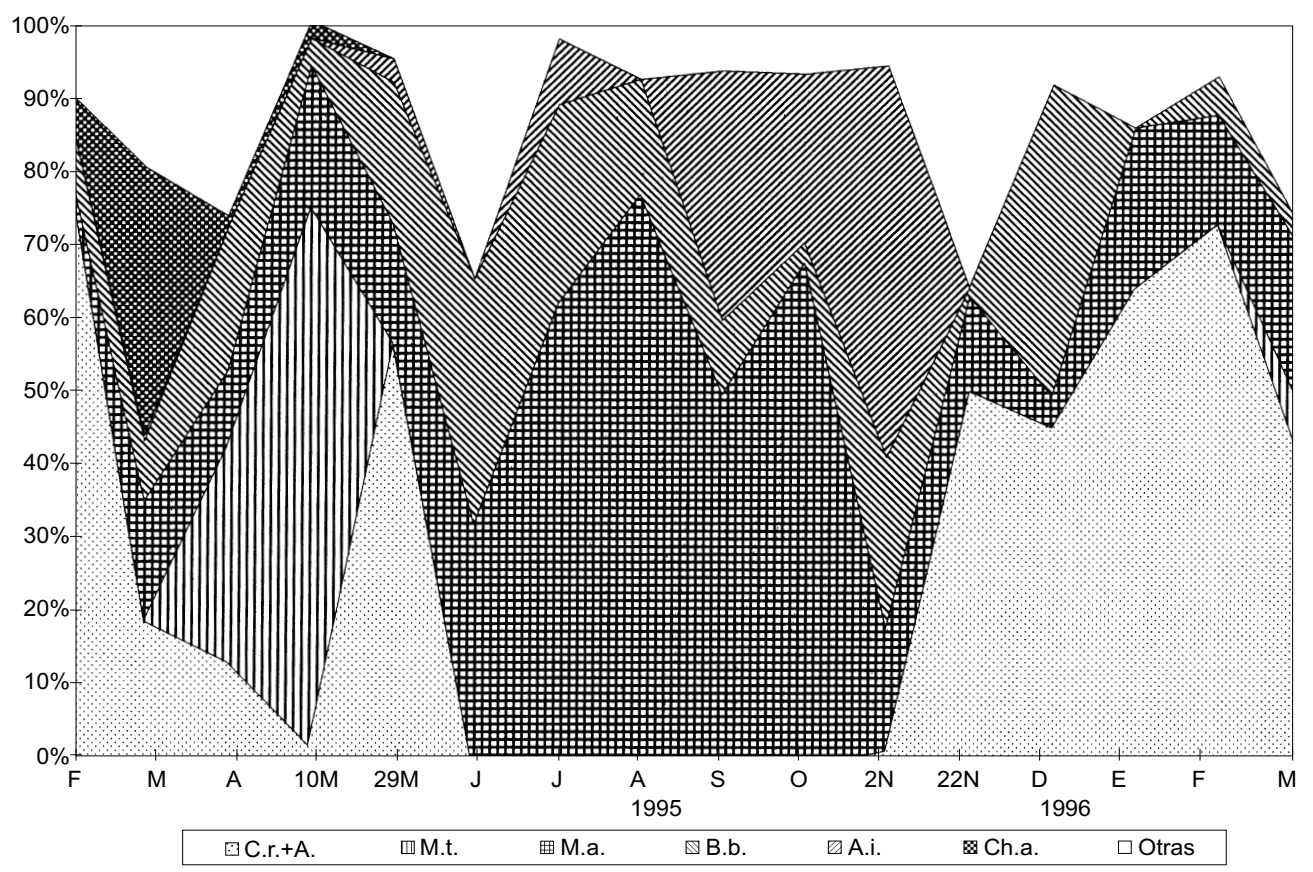

Fig. 10 - Biomasa relativa (\% de la biomasa total) de las especies dominantes del fitoplancton en el estrato próximo a la zona del fondo, en la zona limnética. C.r.+A.: Cylindrospermopsis raciborskii + Aphanizomenon sp., M.t.: Merismopedia tenuissima, M.a.: Microcystis aeruginosa, B.b.: Botryococcus braunii, A.i.: Aulacoseira italica, Ch.a.: Chlorogibba allorgei, Otras: otras especies no dominantes. 
Período A: abarca los dos primeros muestreos (febrero y marzo de 1995) caracterizados por la dominancia de Cylindrospermopsis raciborskii.

Período B: desde abril hasta fines de mayo/ 95, con dominancia de Aphanizomenon sp. y Merismopedia tenuissima.

Período C: desde junio hasta octubre/95, con dominancia de Microcystis aeruginosa y Botryococcus braunii.

Período D: desde noviembre/95 en adelante (hasta finalizar los estudios), con dominancia de Aphanizomenon sp.+Cylindrospermopsis raciborskii.

\section{Período A}

Caracterizó a este período una elevada densidad y biovolumen del fitoplancton. Cylindrospermopsis raciborskii fue la especie dominante el 28-2-95. En el segundo muestreo (el 7-3-95) realizado siete días después y con posterioridad a una tormenta, hubo declinación de la biomasa (Fig. 8) e incremento de la diversidad (Figs. 12 y 13). Cryptomonas ovata y C. erosa fueron las especies dominantes en el estrato superficial, a diferencia del resto de la columna de agua donde predominó $C$. raciborskii a profundidad media y Chlorogibba allorgei en las proximidades del fondo. C. raciborskii se caracterizó por no presentar heterocistes durante todo el período A (en el cual solamente desarrollaron acinetos). La concentración de las diferentes especies de nitrógeno (amonio y nitritos+nitratos) fueron las más elevadas de todo el año (Fig. 15).

\section{Período B}

El 70\% de Cylindrospermopsis raciborskii fue reemplazado por Aphanizomenon sp. + Merismopedia tenuissima. Entre el 16 y 55\% de los filamentos de la primera (Aphanizomenon sp.) se caracterizaron por presentar heterocistes. Hacia el final del período (luego de una lluvia) se produjo el colapso de las poblaciones de $M$. tenuissima, paralelamente a un incremento en la masa de poblaciones de Microcystis aeruginosa y Botryococcus braunii. A su vez, poblaciones de pequeñas algas verdes fueron dominantes en densidad (ind. $\mathrm{mL}^{-1}$ ): Chlorella vulgaris en superficie y pequeños flagelados en profundidad media. La dominancia de estas últimas produjo una disminución de la diversidad (H') basada en la densidad en ese estrato. En cambio la caída de la H' basada en el biovolumen en el fondo se debió al eleva- do volumen de Aphanizomenon sp., M. aeruginosa y B. braunii (Fig. 13).

Las distintas formas de nitrógeno no fueron detectables en algún momento del período B: el amonio al principio y al final y, los nitritos + nitratos, a la inversa (Fig. 15). Paralelamente a estos cambios también se produjeron modificaciones ambientales graduales características de la estación del año, tales como reducción de la temperatura y de la radiación solar (Fig. 4).

\section{Período C}

Hubo una marcada reducción de la densidad y biomasa total del fitoplancton, con dominancia de formas coloniales de Cyanophyta (Microcystis aeruginosa) y Chlorophyta (Botryococcus braunii). Al final del período adquirió importancia también Aulacoseira italica en las proximidades del fondo (Fig. 10). Una excepción se dio al iniciarse el período (muestreo del 14-6-95) en que, en los estratos superior y medio predominaron Aulacoseira italica y Gymnodinium sp., respectivamente (Figs. 8 y 9).

Paralelamente hubo reducción de la temperatura del agua (inferior a $20^{\circ} \mathrm{C}$ ) y de la radiación solar (inferior a $20 \mathrm{MJ} \cdot \mathrm{m}^{-2}$ ). Las precipitaciones fueron escasas (inferior a los $5 \mathrm{~mm}$ ) y días de fuertes vientos (superior a 1,6 m. $\mathrm{s}^{-1}$ ) alternaron con períodos de calma. La $\mathrm{H}^{\text {' }}$ basada en la densidad presentó los valores más elevados de todo el año. La H' basada en el biovolumen fue baja debido a la gran masa alcanzada por grandes colonias de M. aeruginosa y B. braunii.

\section{Período D}

La temperatura y la radiación fueron elevadas. Caracterizó a este período la dominancia de cianofíceas filamentosas heterocistadas Aphanizomenon sp.+Cylindrospermopsis raciborskii. La primera predominó en noviembre y diciembre/95 (hasta el 90\%), mientras que la segunda (C. raciborskii) desde enero/96 (hasta el 60\%) declinando sus poblaciones al final del período. En ambas especies los filamentos con heterocistes representaron entre el 90 y $60 \%$, respectivamente de sus poblaciones (Fig. 15).

\section{d) Variaciones en la composición morfológica}

Las especies dominantes en biomasa durante la mayor parte del año fueron Microcystis aeruginosa y Botryococcus braunii las que alcanzaron mayor masa durante el período C. Se caracterizaron por un gran tamaño (máxima dimensión 
axial linear (MDAL): entre 900 y $1200 \mu \mathrm{m}$ ) y una baja relación area superficial/volumen (AS/V): entre $0,1 \mathrm{y} 0,2 \mu \mathrm{m}^{-1}$. Cylindrospermopsis raciborskii y Aphanizomenon sp. dominantes en masa y densidad en los períodos A, B y D, se caracterizaron por amplias variaciones en la MDAL, con valores entre 180 y $450 \mu \mathrm{m}$, respectivamente y con una relación AS/V de $1,4 \mu \mathrm{m}^{-1}$ durante los períodos de activo crecimiento. Aphanizomenon sp. disminuyó la relación AS/V a $0,7 \mu \mathrm{m}^{-1}$ hacia el final, antes de que se produzca el colapso de sus poblaciones, el 29-5-95.

En los taxones restantes, tales como Aulacoseira italica, Phormidium mucicola, Cryptomonas ovata, C. erosa y Merismopedia tenuissima, la MDAL osciló entre 10 y $389 \mu \mathrm{m}$ y la relación AS/V entre 0,1 y $2,4 \mu \mathrm{m}^{-1}$ (Tabla 1 ).

\section{e) Riqueza específica y diversidad}

La más baja diversidad (H') se registró al inicio de los estudios (28-2-95) en relación a la elevada biomasa de una especie en particular: Cylindrospermopsis raciborskii (28-2-95) en la zona limnética (Fig. 13) y Botryococcus braunii (25-4-95) en la zona litoral (Fig. 14).

En la zona litoral, la $\mathrm{H}^{\text {‘ }}$ basada en el biovolumen, fue mayor que en la zona limnética y osciló entre 1,18 (25-4-95) y 3,11 bits. $\mu \mathrm{m}^{-3}$ (11-3-96) (Fig. 14).

La diversidad basada en la biomasa, en la zona limnética osciló entre 1,04 bits. $\mu \mathrm{m}^{3}$ (28-295) y 2,92 bits $\mu \mathrm{m}^{3}$ (25-4-95), con un patrón de variación semejante en las tres profundidades (Fig. 13). Durante la primera parte del período (entre marzo y junio/95) se mantuvo alta (entre 2,6 y 2,9 bits. $\mu \mathrm{m}^{-3}$ ). A partir de julio presentó una notable caída, manteniéndose durante el resto de los estudios entre 1,9 y 2,4 bits. $\mu m^{-3}$. Esta reducción en la H' se debió fundamentalmente a la presencia de colonias de Microcystis aeruginosa y Botryococcus braunii de gran biovolumen, a las que se sumó Aphanizomenon sp. y Cylindrospermopsis raciborskii a partir del mes de noviembre.

Durante la mayor parte del año la H' siguió un patrón semejante a los valores de equidad. Sin embargo la baja diversidad específica en el muestreo del 22-11-95 en profundidad media, se debió a un escaso número de especies.

La diversidad (H') basada en la densidad, en la zona limnética (Fig. 12) varió entre 0,34 (el
22-11-95) y 3,69 bits.ind $^{-1}$ (el 4-10-95) en el estrato superficial. En el período que va desde fines de mayo hasta principios de noviembre, la $\mathrm{H}^{\prime}$ se mantuvo elevada (con valores superiores a 2,66 bits.ind ${ }^{-1}$ ), con la excepción de los muestreos del 4-10-95 y el 22-11-95, donde la caída de la H' en profundidad media, se debió a la elevada densidad de Sphaerocystis planctonica y Phormidium mucicola, respectivamente. Durante el resto del período, la baja diversidad se correspondió con la dominancia de una o dos especies de cianofíceas. En la zona litoral (de manera similar a lo señalado para la H' basada en la biomasa) también se registraron valores superiores al de la zona limnética (Fig. 14) entre 1,84 (4-1-96) y 4,13 bits.ind ${ }^{-1}$ (2-11-95).

La riqueza específica (RE) o número de especies fue baja durante el período en que dominaron las Cianofíceas filamentosas (Aphanizomenon sp. + Cylindrospermopsis raciborskii), particularmente en las proximidades del fondo, donde se observó la mayor acumulación.

La mayor riqueza específica (RE) se registró en el muestreo del 14-6-95 en que hubo una marcada estratificación de las algas dominantes en biovolumen (Aulacoseira italica, Gymnodinium sp., Botryococcus braunii y Microcystis aeruginosa) y densidad numérica (Chlorella vulgaris y pequeños flagelados verdes). El número de especies de la zona litoral fue muy superior al registrado en la zona limnética de la laguna (Tabla 2).

La zona litoral presentó una significativa correlación linear negativa entre H' y masa del fitoplancton $(r=-0,751 ; p<0,01$; g.l. = 30). En cambio, tal relación no se observó en la zona limnética debido a que altas diversidades ocurrieron durante altas biomasas.

En ambas estaciones de muestreo en cambio, hubo una alta correlación negativa entre abundancia (ind. $\mathrm{mL}^{-1}$ ) y H' basada en la densidad $(\mathrm{r}=-0,751 ; \mathrm{p}<0,01 ; \mathrm{g} .1 .=30)$, excepto en las proximidades del fondo de la zona limnética.

Una ligera diferencia se observó en la H' basada en la densidad, entre la zona litoral y los estratos superficial $(\mathrm{t}=2,651 ; \mathrm{p}<0,02 ; \mathrm{g} .1 .=13)$ e intermedio $(\mathrm{t}=2,235 ; \mathrm{p}<0,05 ;$ g.l. $=13) \mathrm{de}$ la zona limnética.

En ambas estaciones de muestreo la H' basada en la densidad se correlacionó negativamente con la abundancia y positivamente con la riqueza específica $(\mathrm{r}=0,703 ; \mathrm{p}<0,01 ;$ g.l. $=30)$. 


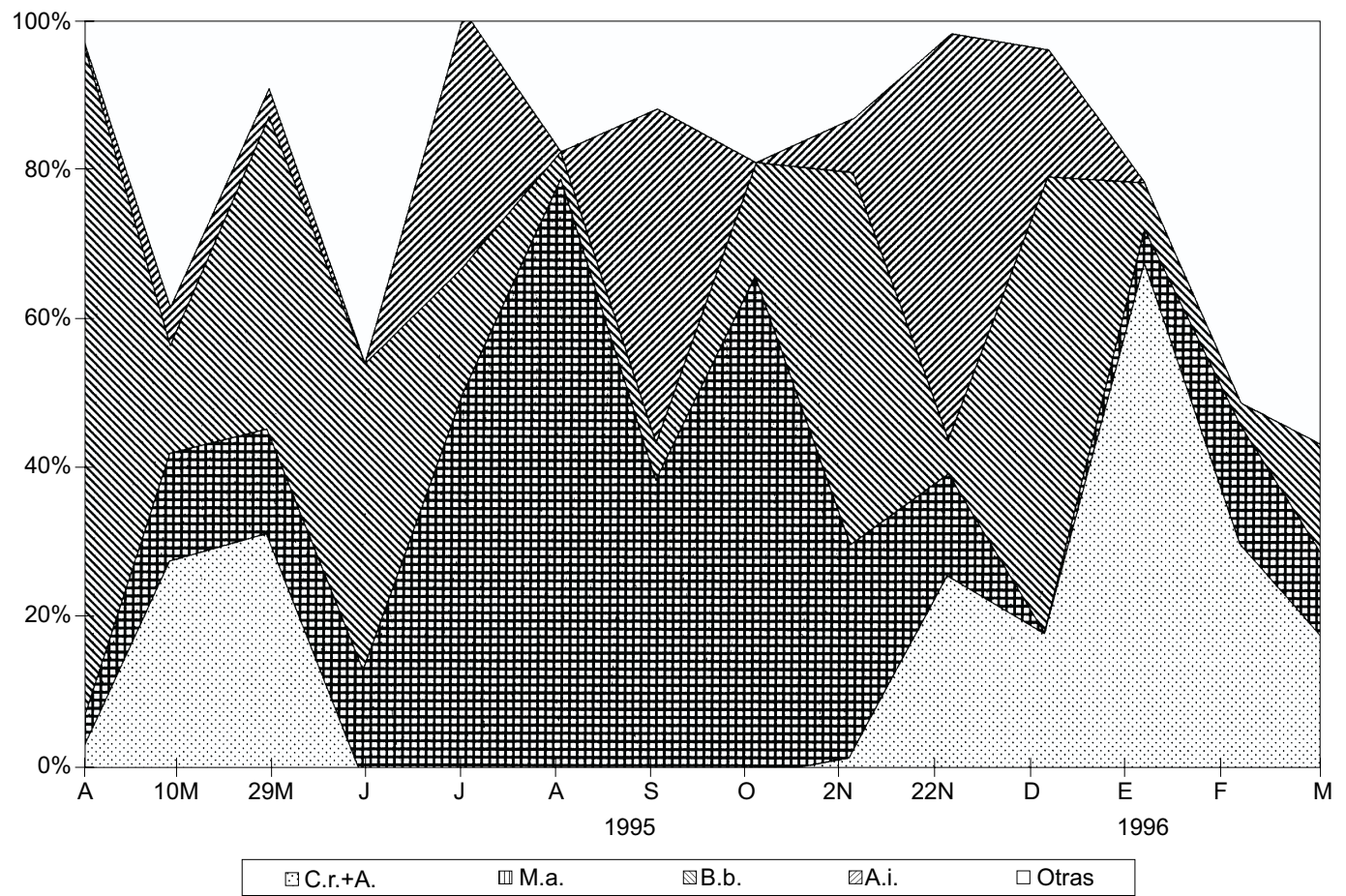

Fig. 11 - Variación temporal de la biomasa relativa (\%) de las especies dominantes del fitoplancton en la zona litoral del lago. C.r. + A.: Cylindrospermopsis raciborskii + Aphanizomenon, M.a.: Microcystis aeruginosa, B.b.: Botryococcus braunii, A.i.: Aulacoseira italica, Otras: otras especies no dominantes.

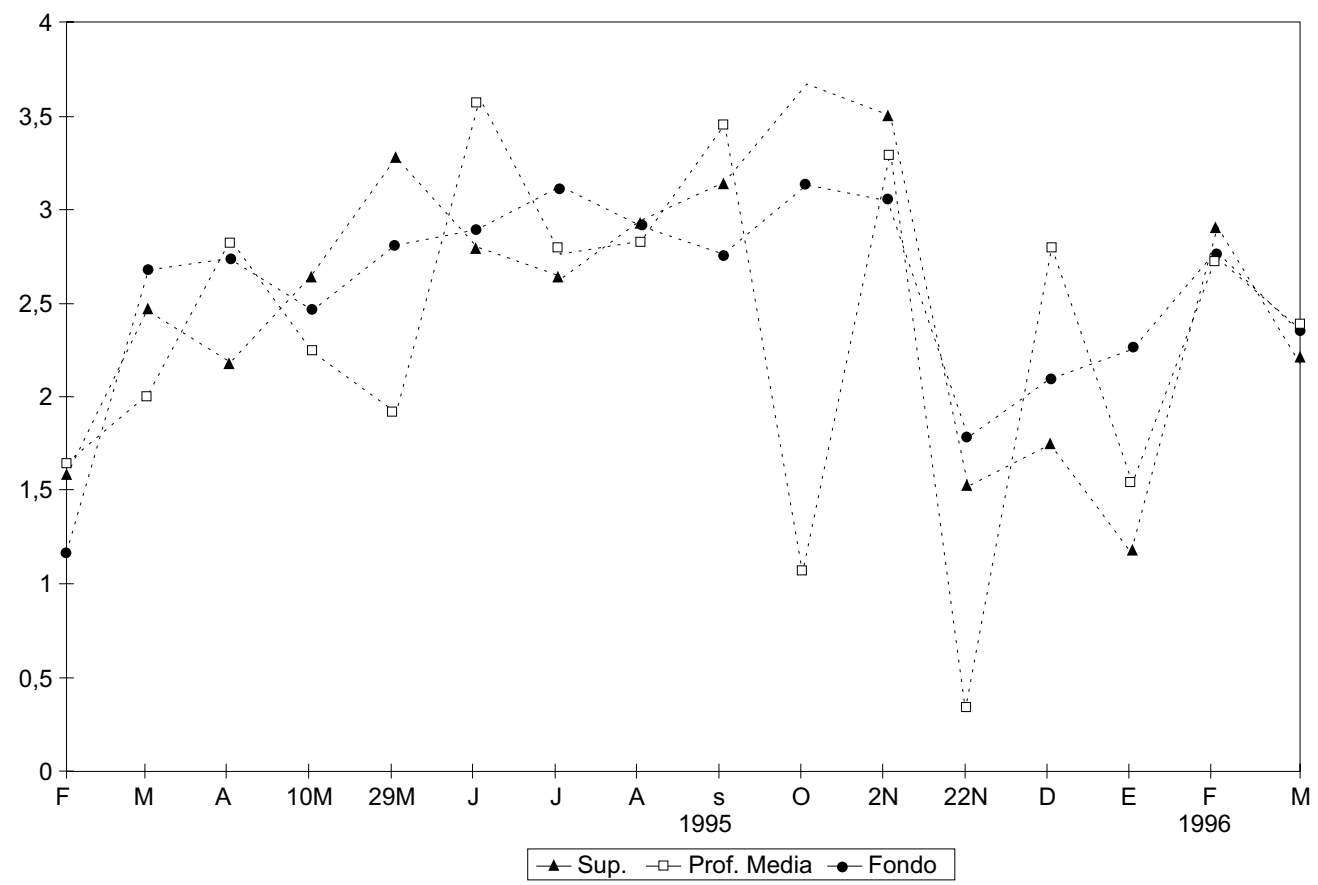

Fig. 12 - Variación de la diversidad del fitoplancton basada en la densidad (bits.ind ${ }^{-1}$ ) a diferentes profundidades de la zona limnética del lago. 


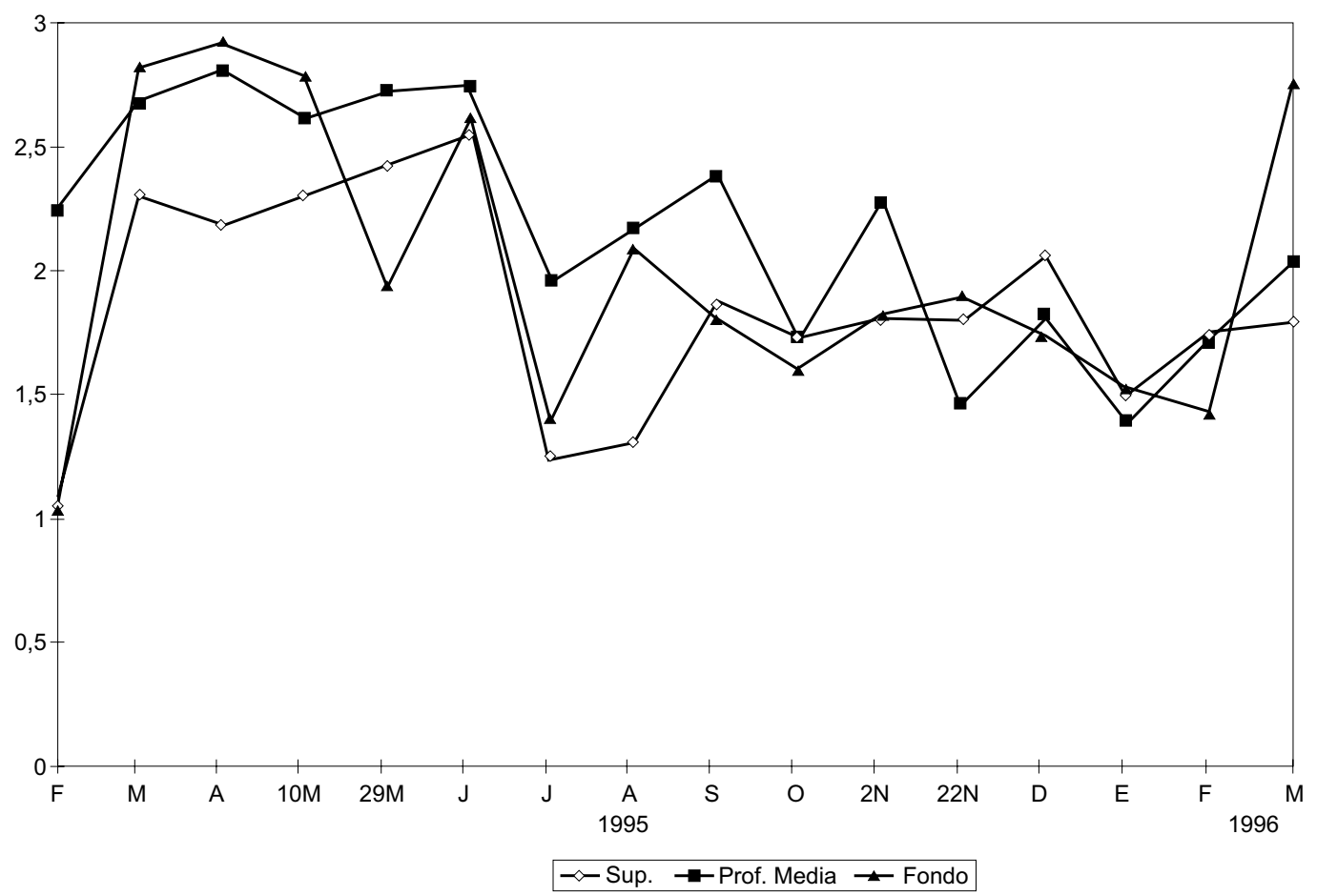

Fig. 13 - Variación de la diversidad del fitoplancton basada en el biovolumen (bits. $\mu \mathrm{m}^{-3}$ ) a diferentes profundidades de la zona limnética.

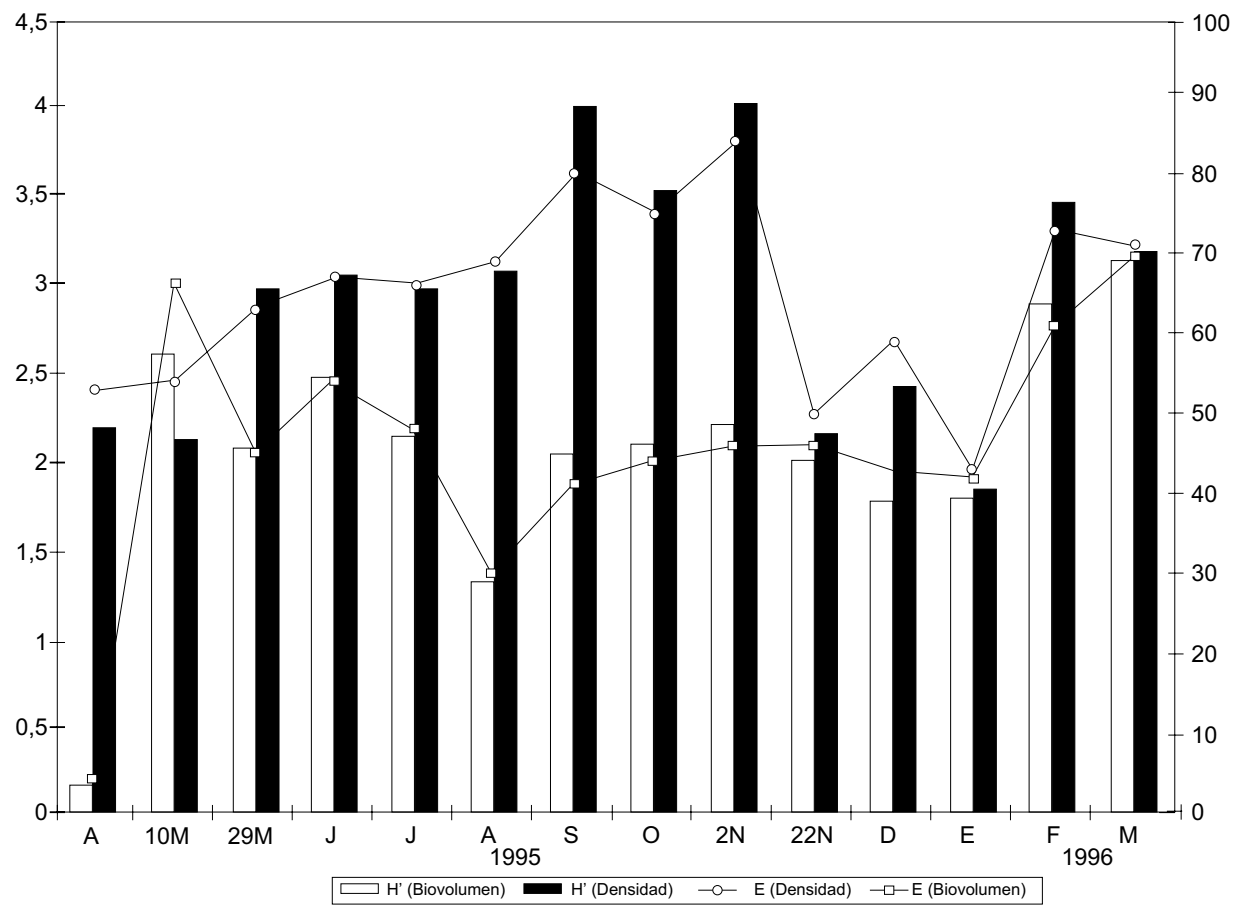

Fig. 14 - Variaciones de la diversidad (basada en la densidad y el biovolumen) y de la equidad en la zona litoral de la "laguna Aeroclub". 
TABLA 1

Características morfológicas de las especies dominantes en términos de biomasa.

Forma: formas geométricas. V: volumen celular $\left(\mu \mathrm{m}^{3}\right), \mathrm{AS}$ : área superficial $\left(\mu \mathrm{m}^{2}\right)$, AS/V: relación área superficial/volumen $\left(\mu \mathrm{m}^{-1}\right)$, MDAL: máxima dimensión axial linear $(\mu \mathrm{m})$.

\begin{tabular}{|c|c|c|c|c|c|}
\hline Especies & Forma & $\mathbf{V}\left(\mu \mathbf{m}^{3}\right)$ & $\operatorname{AS}\left(\mu \mathbf{m}^{2}\right)$ & $\mathbf{A S} / \mathbf{V}\left(\mu \mathbf{m}^{-1}\right)$ & MDAL $(\mu \mathrm{m})$ \\
\hline O Anabaena perturbata $*$ & elipsoide & 5650 & 5650 & 1 & 200 \\
\hline - Aphanizomenon sp. ** & cilindro & 5914 & 8539 & 1,4 & 450 \\
\hline - Cylindrospermopsis raciborskii ** & cilindro & 371 & 260 & 0.7 & 180 \\
\hline - Merismopedia tenuissima $*$ & esfera & 768 & 1248 & 1.6 & 30 \\
\hline - Microcystis aeruginosa * & esfera & $2,5 \times 10^{6}$ & $4,5 \times 10^{6}$ & 0,2 & 1200 \\
\hline - Phormidium mucicola $* *$ & cilindro & 53 & 130 & 2,4 & 10 \\
\hline O Planitolyngbya subtiiis $* *$ & cilindro & 368 & 741 & 2 & 117 \\
\hline - Botryococcus braunii ** & esfera & $18,8 \times 10^{6}$ & $2,5 \times 10^{6}$ & 0,1 & 900 \\
\hline O Chlorella vulgaris & esfera & 113 & 113 & 1 & 6 \\
\hline О Pequeños flagelados verdes & elipsoide & 54 & 13 & 0,2 & 6,5 \\
\hline O Monoraphidium contortum & bicono & 20 & 34 & 1,7 & 12 \\
\hline O Monoraphidium tortile & bicono & 22 & 44 & 2 & 17 \\
\hline O Sphaerocystis planctonica * & esfera & 272 & 402 & 1,5 & 24 \\
\hline - Aulacoseira italica * & cilindro & 43424 & 14480 & 0,3 & 389 \\
\hline ○ Chlorogibba allorgei & esfera & 697 & 380 & 0,5 & 11 \\
\hline - Cryptomonas ovata & cono & 888 & 90 & 0,1 & 18 \\
\hline - Cryptomonas erosa & elipsoide & 559 & 125 & 0,2 & 17 \\
\hline - Gymnodinium sp. & esfera & 1767 & 707 & 0,4 & 15 \\
\hline O Trachelomonas volvocina & esfera & 905 & 4520 & 0,5 & 12 \\
\hline
\end{tabular}

- especies dominantes en biovolumen.

O especies dominantes en densidad.

- especies dominantes en densidad y biovolumen.

En las especies señaladas con un asterisco (*) los biovolumenes fueron calculados a partir de células individuales. Las señaladas con doble asterisco $(* *)$ corresponden a formas filamentosas o coloniales donde no fue posible distinguir células individuales, tomándose como referencia la longitud de los filamentos o el diámetro de las colonias.

\section{f) Clorofila a}

La clorofila $a$ (Fig. 4) de un modo general reflejó la variación anual de la biomasa del fitoplancton (correlación linear, $r=0,702 ; \mathrm{p}<0,01$ ), oscilando entre valores de 0,7 (agosto de 95) y $8 \mu \mathrm{g} . \mathrm{L}^{-1}$ (el 10-05-95).

\section{DISCUSION Y CONCLUSIONES}

En la "Laguna Aeroclub" se registraron 137 taxones de algas, sin embargo fueron pocas las especies que se caracterizaron por su dominancia (Tabla 1). Cyanophyta y Chlorophyta fueron los grupos que predominaron a lo largo del año, de manera similar a lo observado años anteriores en éste ambiente (Matveev et al., 1992) y en otros de características limnológicas semejantes (Bonetto et al., 1978a, 1978b) durante primavera y verano.
La "Laguna Aeroclub" presenta características eutróficas, tanto por la elevada densidad del fitoplancton como por la composición de los taxones que lo integran, caracterizado por un reducido número de especies dominantes (principalmente cianofíceas) durante los meses más cálidos del año.

La abundancia del fitoplancton y las concentraciones de clorofila $a$ fueron elevadas (máximos de 9689 ind. $\mathrm{mL}^{-1}$ y $8 \mu \mathrm{g} . \mathrm{L}^{-1}$, respectivamente). Tales valores fueron similares o superiores a lo registrado en otros ambientes lénticos de América del Sur, en clima tropical y subtropical (Schmidt, 1970; Bonetto et al., 1978a, 1978b; Huszar, 1989, Marinho et al., 1990, 1993; Giani y Leonardo, 1988; García de Emiliani, 1980, 1993; Zalocar de Domitrovic, 1990, 1992, 1993). 
La menor densidad y biomasa de la zona litoral (en relación a la zona limnética) coincide con observaciones señaladas en otro tipo de ambientes vegetados de la región argentina (Zalocar de Domitrovic, 1992; Zalocar de Domitrovic et al., 1986). La escasa densidad y biomasa algal de la zona litoral (máximos de 3470 ind. $\mathrm{mL}^{-1}$ y $4,4 \times 10^{6} \mu \mathrm{m}^{3} . \mathrm{L}^{-1}$, respectivamente) con respecto al área limnética probablemente se deba a una fuerte competición entre el fitoplancton y los macrófitos, por nutrientes similares (Oleksowicz, 1987). Excepcionalmente la elevada biomasa registrada en la zona litoral $\left(33 \times 10^{6} \mu \mathrm{m}^{3} . \mathrm{L}^{-1}\right)$ en un único muestreo, se debió a la acumulación de colonias de Microcystis aeruginosa y Botryococcus braunii en la dirección de los vientos predominantes en ese momento.

Varios autores (entre ellos Hutchinson, 1967, Wetzel, 1981, Esteves, 1988) señalan que el reciclado de nutrientes y el flujo de energía en la zona litoral es controlado por la comunidad de macrófitos, mientras que en la zona limnética lo es por el fitoplancton. La zona litoral (compara- tivamente con la zona limnética) presentó una elevada diversidad y riqueza específica. En la zona litoral se registraron 125 especies contra 86 de la zona limnética, mientras que 74 fueron comunes a ambas. Las condiciones físico-químicas diferentes a la zona limnética y la mayor variedad de nichos que ofrecen los macrófitos acuáticos de la zona litoral serían propicios para una mayor diversidad específica.

Utilizando la terminología de Reynolds (1988), pudieron identificarse diferentes tipos de estrategas. En general, predominaron en biomasa los estrategas S tales como colonias de Microcystis aeruginosa (Cyanophyta) y Botryococccus braunii (Chlorophyta). Formas filamentosas de Cyanophyta: Cylindrospermopsis raciborskii y Aphanizomenon sp. predominaron en densidad y biomasa durante los meses más cálidos del año (en primavera, verano y parte del otoño) con temperaturas y radiación superiores a $20^{\circ} \mathrm{C}$ y $20 \mathrm{MJ} . \mathrm{cm}^{-2}$, respectivamente. Entre fines de mayo y principios de noviembre (período con predominio de vientos) fueron dominantes en densidad (pero no

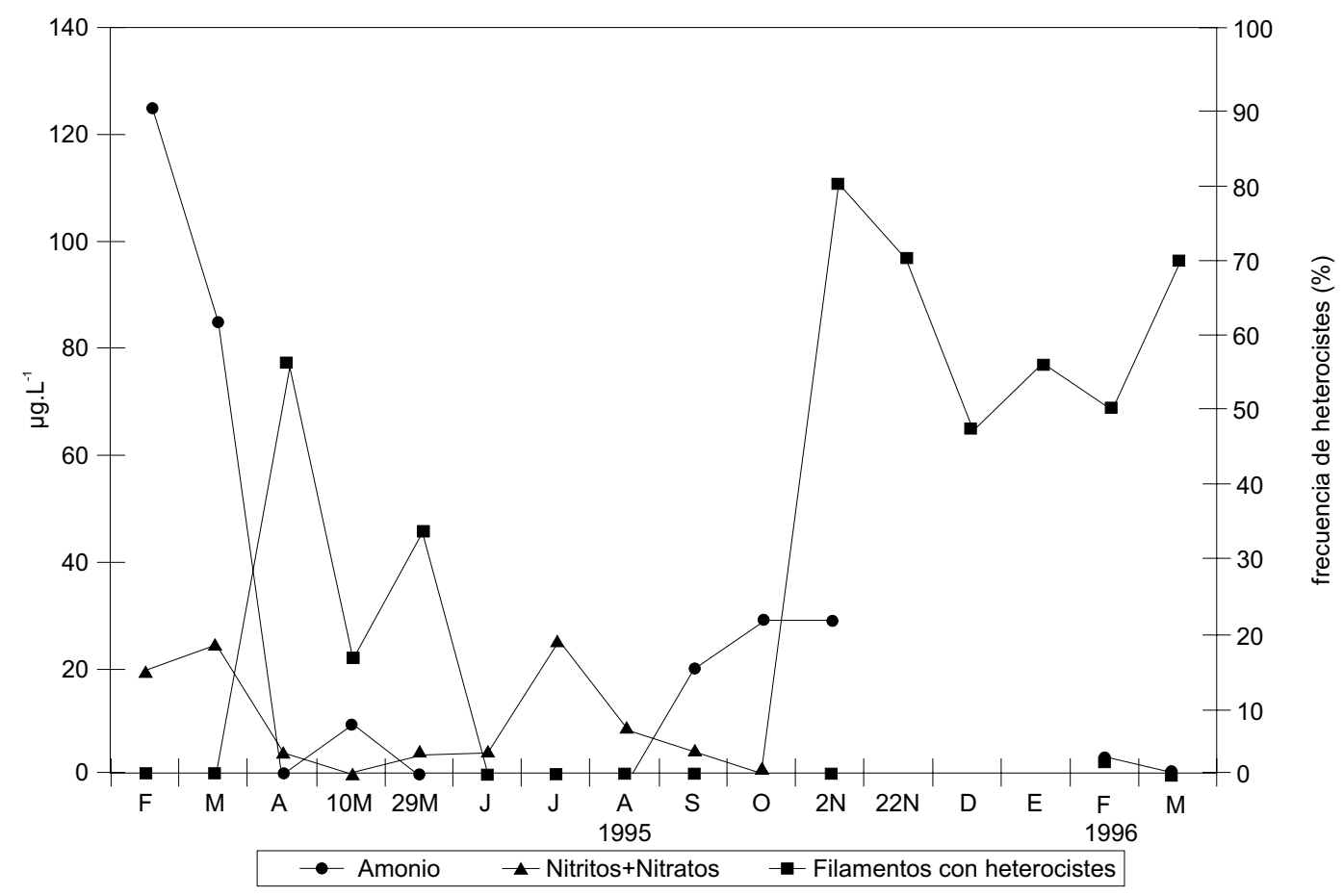

Fig.15 - Variación de las concentraciones de las principales especies de nitrógeno (en $\mu \mathrm{g} . \mathrm{L}^{-1}$ ) en relación a la frecuencia de filamentos con heterocistes de Cylindrospermopsis raciborskii, Aphanizomenon sp. y Anabaena perturbata 
en biovolumen) estrategas $\mathrm{C}$, es decir algas pequeñas con una alta relación AS/V tales como Chlorella vulgaris, pequeños flagelados verdes, Monoraphidium contortum, M. tortile, Phormidium mucicola, Cryptomonas ovata y C. erosa. Ocasionalmente y durante los períodos de mayor disturbio (ocasionados principalmente por el viento) se observaron estrategas $\mathrm{R}$ (Aulacoseira italica y Gymnodinium sp.).

La declinación de las poblaciones de Cylindrospermopsis raciborskii y Aphanizomenon sp. se produjeron paralelamente a una reducción de la radiación solar otoñal, entre 15 y $20{\mathrm{MJ} . \mathrm{m}^{-2}}^{-2}$ (Fig. 4) y de la temperatura del agua (inferior a $20^{\circ} \mathrm{C}$ ). Estas especies se mantuvieron como un inóculo potencial (filamentos y/o acinetos) sobre los sedimentos del fondo durante su ausencia en la columna de agua (desde junio hasta noviembre). Las mismas reiniciaron su crecimiento cuando la radiación solar (a fines de primavera) superó $\operatorname{los} 20 \mathrm{MJ} \cdot \mathrm{m}^{-2}$ y la temperatura del agua fue superior a $\operatorname{los} 20^{\circ} \mathrm{C}$ en las proximidades del fondo.

Varios autores han señalado acerca de la importancia del rol que juega la inoculación directa de las algas desde el bentos (ej. Aulacoseira, cianofíceas etc.). La permanencia de las algas azules sobre los sedimentos del fondo durante una parte de su ciclo de vida, antes de su rápida aparición en la columna de agua fue señalada para numerosos ambientes por Sirenko et al. (1969), Preston et al. (1980), Barbiero \& Welch (1992) Barbiero \& Kann (1994).

En la "laguna Aeroclub" el incremento de la luz y de la temperatura que precedieron al episodio del crecimiento de las poblaciones de Aphanizomenon sp. y Cylindrospermopsis raciborskii en noviembre de 1995, coincide con observaciones señaladas para Aphanizomenon flos-aquae y otras especies de cianofíceas por Reynolds (1972); Barbiero (1993) y Barbiero \& Kann (1994.). Estos autores sugieren que la luz es uno de los factores más importantes en la determinación del tiempo y magnitud de la resuspensión de algunas especies de cianofíceas (tales como Anabaena circinalis, Gloeotrichia echinulata y Aphanizomenon flos-aquae).

En la "laguna Aeroclub", Microcystis aeruginosa (a diferencia de Aphanizomenon sp. y $C$. raciborskii) se registró en el plancton durante todo el año con mayor densidad y biomasa en las proximidades del fondo.
La concentración de fósforo (ortofosfatos) no fue detectable en el estrato superficial. Es de suponer que el crecimiento del fitoplancton fue mantenido principalmente por los nutrientes contenidos en los sedimentos del fondo y probablemente también por translocación de pequeñas cantidades desde los sedimentos y liberados a través de diferentes mecanismos hacia la columna de agua, o por intermedio de algas meroplanctónicas.

Hay evidencias de que muchas especies de cianofíceas (al igual que otros grupos de algas) pueden sobrellevar un período de asimilación de nutrientes mientras están en contacto con la gran reserva de $\mathrm{P}$ del sedimento intersticial y subsecuentemente suspenderse en la columna de agua con suficientes reservas como para salvar una futura captación durante numerosas divisiones celulares. Lo señalado quizás estaría relacionado a las cianofíceas registradas en la "laguna Aeroclub", tales como Anabaena perturbata que hizo su aparición en la columna de agua en septiembre-octubre pasando un período de su vida en el fondo como acinetos, al igual que Aphanizomenon sp. y Cylindrospermopsis raciborskii, como filamentos y/o acinetos y también Microcystis aeruginosa como colonias (de observaciones realizadas en muestras de sedimento superficial del fondo). Si bien la última especie no desapareció de la columna de agua durante todo el año, su mayor abundancia y biomasa se registró en las proximidades del fondo, la cual captaría P no sólo de los sedimentos, sino también el poco $\mathrm{P}$ disponible de toda la columna de agua.

La limitación del $P$ en éste ambiente fue demostrada por experiencias de laboratorio a la que al agua del estrato superficial se le adicionó nutrientes inorgánicos: amonio, nitratos y fosfatos, en una relación N/P de 10:1 (Zalocar de Domitrovic et al., en prep.).

En la "laguna Aeroclub" se registraron especies de cianofíceas fijadoras y no fijadoras de nitrógeno. Entre las especies heterocistadas (fijadoras de nitrógeno) se observó Anabaena perturbata, Cylindrospermopsis raciborskii y Aphanizomenon sp. La primera sólo se registró en los muestreos de septiembre y octubre (en este último en mayor densidad). C. raciborskii no desarrolló heterocistes en el período A, cuando las concentraciones de diferentes especies de $\mathrm{N}$ fueron las más altas del año, período en que se 
produjeron las mayores precipitaciones pluviales. En cambio, durante el resto de los períodos siempre desarrollaron heterocistes, al igual que Aphanizomenon sp. El resto de las especies de cianofíceas no heterocistadas correspondieron a Microcystis aeruginosa, Merismopedia tenuissima y Aphanocapsa spp. entre otros taxones escasamente representados en biomasa.

De manera similar a lo que fuera señalado por Blomqvist et al. (1994) para lagos eutróficos del hemisferio norte, es de suponer que cuando se agota el pool de $\mathrm{N}$ inorgánico, la recirculación de $\mathrm{N}$ orgánico es suficiente como para sustentar la productividad en toda la columna de agua. En cambio en situaciones de estratificación (cuando la recirculación no es lo suficientemente alta co- mo para sustentar la productividad), aparecerían, y/o desarrollarían heterocistes, las especies fijadoras de nitrógeno.

En la "laguna Aeroclub" la frecuencia de cianofíceas filamentosas con heterocistes estuvo inversamente correlacionada con las concentraciones de nitritos + nitratos $(\mathrm{r}=-0,767 ; \mathrm{p}<0,01$; g.l. $=$ 14) y de amonio $(r=-0,674 ; p<0,01 ;$ g.l. $=14)$. A su vez las concentraciones de las diferentes especies de nitrógeno estuvieron fuertemente correlacionadas con el valor medio diario de las lluvias caídas 10 días antes de cada muestreo: $r=0,951$; $\mathrm{p}<0,01$ (amonio) y $\mathrm{r}=0,940 ; \mathrm{p}<0,01$ (nitritos + nitratos). Una alta correlación positiva se registró entre las masa del fitoplancton y las precipitaciones caídas $(r=0,772 ; p<0,01 ; g .1=30)$.

TABLA 2

Lista de las especies de algas registradas en la "laguna Aeroclub". Estación A: zona limnética, Estación B: zona litoral, x: presencia, -: ausencia, N.I.: no identificado.

\begin{tabular}{|c|c|c|}
\hline & Estación A & Estación B \\
\hline \multicolumn{3}{|l|}{ CYANOPHYCEAE } \\
\hline Anabaena aphanizomenoides Forti & $\mathrm{X}$ & $\mathrm{X}$ \\
\hline A. perturbata Hill. & $\mathrm{X}$ & $\mathrm{X}$ \\
\hline A. spiroides $\mathrm{Kleb}$. & $\mathrm{X}$ & - \\
\hline Aphanizomenon sp. & $\mathrm{X}$ & $\mathrm{X}$ \\
\hline A. elachista W. \& G. S. West var. elachista & $\mathrm{X}$ & $\mathrm{X}$ \\
\hline A. elachista var. planctonica G. M. Smith & $\mathrm{X}$ & - \\
\hline A. pulchra (Kütz.) Rabenh. & $\mathrm{X}$ & $\mathrm{X}$ \\
\hline A. roeseana de Bary & - & $\mathrm{X}$ \\
\hline Aphanothece stagnina (Spreng.) A. Br. & - & $\mathrm{X}$ \\
\hline Coelosphaerium kuetzingianum Nag. & $\mathrm{X}$ & $\mathrm{X}$ \\
\hline Cylindrospermopsis raciborskii (Wolosz.) Seenayya et Subba Raju & $\mathrm{X}$ & $\mathrm{X}$ \\
\hline Gomphosphaeria aponina Kütz. & $\mathrm{X}$ & $\mathrm{X}$ \\
\hline G. Iacustris Chodat & - & $\mathrm{X}$ \\
\hline Lyngbya gracilis Rabenh. & - & $\mathrm{X}$ \\
\hline Merismopedia tenuissima Lemm. & $\mathrm{X}$ & $\mathrm{X}$ \\
\hline Microcystis aeruginosa Kütz. & $\mathrm{X}$ & $\mathrm{X}$ \\
\hline M. flos-aquae (Wittr.) Kirchner & $\mathrm{X}$ & $\mathrm{X}$ \\
\hline Oscillatoria sp. & - & $\mathrm{X}$ \\
\hline Phormidium mucicola Hüb.-Pest. \& Naum. & $\mathrm{X}$ & $\mathrm{X}$ \\
\hline Planktolyngbya conforta (Lemm.) Anag. \& Kom. & $\mathrm{X}$ & $\mathrm{X}$ \\
\hline P. subtilis (W. West) Anag. \&Kom. & $\mathrm{X}$ & $\mathrm{X}$ \\
\hline \multicolumn{3}{|l|}{ CHLOROPHYCEAE } \\
\hline Actinastrum hantzschii Lagerh. & $\mathrm{X}$ & - \\
\hline Ankistrodesmus falcatus (Corda) Ralfs & $\mathrm{X}$ & $\mathrm{X}$ \\
\hline
\end{tabular}


Estación A

Estación B

\section{CHLOROPHYCEAE (cont.)}

A. arcuatus Kors.

A. bibraianus (Reinsch) Kors. Ankyra judayi (G. M. Smith) Fott

Botryococcus braunii Kütz.

Carteria sp.

Chlamydomonas sp.

Chlorella vulgaris Beij.

Chlorogonium sp.

Choricystis coccoides (Rodhe \& Skuja) Fott

Closteriopsis acicularis (G. M. Smith) Belch \& Swale

Coelastrum indicum Turn.

C. pseudomicroporum Kors.

C. pulchrum Schmidle

Coenococcus planctonicus Kors.

Crucigenia tetrapedia (Kirchn.) W. \& G. S. West

C. quadrata Morr.

Dictyosphaerium ehrenbergianum Näg.

Dimorphococcus lunatus A. Br.

D. cecoalensis Tell

Elakatothrix gelatinosa Wille

Eudorina elegans Ehr.

Flagelado N. I.

Kirchneriella lunaris (Kirchn.) Moeb.

Monoraphidium arcuatum (Kors.) Hind.

M. contortum (Thur.) Kom.-Legn.

M. convolutum (Corda) Kom.-Legn.

M. griffithii (Berk.) Kom.-Legn.

M. pusillum (Printz) Kom.-Legn.

M. tortile (W. \& G. S. West) Kom. -Legn.

Nephrocytium agardhianum Näg.

Oocystis lacustris Chod.

Pediastrum duplex Meyen

P. simplex Meyen

P. tetras (Ehr.) Ralfs

Scenedesmus sp.

S. caribeanus Kom.

S. oahuensis (Lemm.) G. M. Smith

S. perforatus Lemm.

S. protuberans Fritsch

S. quadricuada(Turp.) Bréb.

S. smithii Teil.

Schroederia setigera (Schrod.) Lemm.

Sphaerocystis planctonica (Kors.) Bourr.

S. polycocca Kors.

S. schroeteri Schod.

X

$-$

X

$\mathrm{X}$

X

$\mathrm{X}$

$\mathrm{X}$

$-$

X

-

X

$-$

$-$

-

$\mathrm{X}$

$\mathrm{X}$

- X

- X

- X

- $\mathrm{X}$

X X

- X

$\begin{array}{ll}x & X \\ X & \end{array}$

X X

- X

X -

X $\quad X$

X $\mathrm{X}$

- X

- X

X $\quad X$

- X

$\begin{array}{ll}X & X\end{array}$

X $\quad \mathrm{X}$

- X

- X

X X

$\begin{array}{lll}- & \\ - & X\end{array}$

$\mathrm{X} \quad \mathrm{X}$

- $\quad X$

$\mathrm{X} \quad \mathrm{X}$

$\mathrm{X} \quad \mathrm{X}$

- $\quad X$

X $\quad \mathrm{X}$ 
TABLA 2 (continuación)

\begin{tabular}{|c|c|c|}
\hline & Estación A & Estación B \\
\hline \multicolumn{3}{|l|}{ ULOTHRICOPHYCEAE } \\
\hline Oedogonium sp. 1 & - & $\mathrm{X}$ \\
\hline Oedogonium sp. 2 & - & $\mathrm{X}$ \\
\hline Radiofilum transversalis (Bréb.) Ramanathan & $\mathrm{X}$ & $\mathrm{X}$ \\
\hline Ulothrix bipyrenoidosa Fritsch et Rich & - & $\mathrm{X}$ \\
\hline \multicolumn{3}{|l|}{ CONJUGATOPHYCEAE } \\
\hline Closterium sp. & - & $\mathrm{X}$ \\
\hline C. ehrenbergii Menegh. & $\mathrm{X}$ & $\mathrm{X}$ \\
\hline C. gracile Bréb. & - & $\mathrm{X}$ \\
\hline Cosmarium blyttii Wille & $\mathrm{X}$ & $\mathrm{X}$ \\
\hline C. paraguayense Borge & - & $\mathrm{X}$ \\
\hline C. pusillum (Bréb.) Arch. & $\mathrm{X}$ & $\mathrm{X}$ \\
\hline Cosmocladium constrictum Arch. & - & $\mathrm{X}$ \\
\hline Euastrum abruptum Nordst. & - & $\mathrm{X}$ \\
\hline E. evolutum (Nordst.) West et West & - & $\mathrm{X}$ \\
\hline E. evolutum var. nycticorax Couté et Tell & - & $\mathrm{X}$ \\
\hline Hyalotheca siolii (Forst.) Forst. & - & $\mathrm{X}$ \\
\hline Micrasterias radiata Hass. & - & $\mathrm{X}$ \\
\hline Sphaerozosma laeve var. Iatum (W. et G. S. West) Forst. & - & $\mathrm{X}$ \\
\hline Spirogyra sp. & - & $\mathrm{X}$ \\
\hline Spondylosium pulchrum (Bail.) Archer & - & $\mathrm{X}$ \\
\hline Staurastrum bieneanum var. depressum Messik. & - & $\mathrm{X}$ \\
\hline S. brasiliense Nordst. & - & $\mathrm{X}$ \\
\hline S. leptacanthum var. borgei Forst. & - & $\mathrm{X}$ \\
\hline S. leptocladum var. cornutum Wille & - & $\mathrm{X}$ \\
\hline S. leptocladum var. subinsigne Scott \& Gronbl. & - & $\mathrm{X}$ \\
\hline S. pseudosebaldi Wille & $\mathrm{X}$ & $\mathrm{X}$ \\
\hline S. rotula Nordst. & - & $\mathrm{X}$ \\
\hline S. setigerum Cleve & $X$ & $\mathrm{X}$ \\
\hline Staurodesmus lobatus var. ellipticus fa. minor (Smith) Teil. & $\mathrm{X}$ & $\mathrm{X}$ \\
\hline S. triangularis(Lagerh.) Teil. & $\mathrm{X}$ & $\mathrm{X}$ \\
\hline Teilingia granulata (Roy et Biss.) Bourr. & - & $\mathrm{X}$ \\
\hline \multicolumn{3}{|l|}{ BACILLARIOPHYCEAE } \\
\hline Aulacoseira granulata (Ehr.) Sirnonsen & $\mathrm{X}$ & $\mathrm{X}$ \\
\hline A. italica (Ehr.) Simonsen & $\mathrm{X}$ & $\mathrm{X}$ \\
\hline Eunotia indica Grun. & - & $\mathrm{X}$ \\
\hline Nitzschia amphibia Grun. & $\mathrm{X}$ & $\mathrm{X}$ \\
\hline Rhizozolenia eriensis H. L. Smith & $\mathrm{X}$ & - \\
\hline Synedra ulna (Nitzsch) Ehr. & $\mathrm{X}$ & $\mathrm{X}$ \\
\hline \multicolumn{3}{|l|}{ CHRYSOPHYCEAE } \\
\hline Dinobryon sertularia Ehr. & $\mathrm{X}$ & $\mathrm{X}$ \\
\hline Mallomonas sp. 1 & $\mathrm{X}$ & $\mathrm{X}$ \\
\hline Mallomonas sp. 2 & $\mathrm{X}$ & $\mathrm{X}$ \\
\hline Synura sp. & $\mathrm{X}$ & $\mathrm{X}$ \\
\hline
\end{tabular}


TABLA 2 (continuación)

\begin{tabular}{|c|c|c|}
\hline & Estación A & Estación $\mathrm{B}$ \\
\hline \multicolumn{3}{|l|}{ XАNTHOPHYCEAE } \\
\hline Chlorogibba allorgei Bourr. & $\mathrm{x}$ & $\mathrm{x}$ \\
\hline Goniochloris sculpta Geitler & $\mathrm{X}$ & $\mathrm{x}$ \\
\hline Polyedriella sp. & $\mathrm{X}$ & $\mathrm{x}$ \\
\hline Pseudostaurastrum limneticum (Borge) Chodat & $\mathrm{x}$ & $\mathrm{x}$ \\
\hline P. Iobulatum (Nag.) Chodat & $\mathrm{x}$ & $\mathrm{x}$ \\
\hline Tetraedriella jovetii (Bourr.) Bourr. & $\mathrm{X}$ & $\mathrm{x}$ \\
\hline Tetraplektron torsum (Skuja) Dedus. & $\mathrm{x}$ & $\mathrm{x}$ \\
\hline Trachydiscus minutus (Bourr.) Ettl & $\mathrm{x}$ & $\mathrm{x}$ \\
\hline \multicolumn{3}{|l|}{ EUGLENOPHYCEAE } \\
\hline Euglena sp. & $\mathrm{x}$ & $\mathrm{x}$ \\
\hline E. acus Ehr. & - & $\mathrm{x}$ \\
\hline E. oxyuris Schmarda & $\mathrm{X}$ & - \\
\hline E. polymorpha Dang. & $\mathrm{x}$ & $\mathrm{x}$ \\
\hline Lepocinclis salina Fritsch & $\mathrm{X}$ & - \\
\hline Phacus sp. & $\mathrm{x}$ & - \\
\hline P. platalea Drez. & $\mathrm{X}$ & $\mathrm{x}$ \\
\hline Strombomonas ovalis (Playf.) Defl. & $\mathrm{x}$ & - \\
\hline Trachelomonas armata (Ehr.) Stein. & $\mathrm{X}$ & $\mathrm{x}$ \\
\hline T. armata var. steinii Lemm & $\mathrm{x}$ & $\mathrm{x}$ \\
\hline T. volvocina Ehr. & $\mathrm{X}$ & $\mathrm{X}$ \\
\hline \multicolumn{3}{|l|}{ CRYTOPHYCEAE } \\
\hline Chroomonas acusa Uterm. & $\mathrm{x}$ & $\mathrm{x}$ \\
\hline Cryptomonas erosa Ehr. & $\mathrm{X}$ & $\mathrm{x}$ \\
\hline C. marssonii Skuja & $\mathrm{x}$ & $\mathrm{x}$ \\
\hline C. ovata Ehr. & $\mathrm{X}$ & $\mathrm{x}$ \\
\hline C. pusilla Bachman & $\mathrm{x}$ & - \\
\hline C. rostratiformis Skuja & $\mathrm{x}$ & $\mathrm{x}$ \\
\hline Rhodomonas sp. & $\mathrm{X}$ & $\mathrm{X}$ \\
\hline \multicolumn{3}{|l|}{ DINOPHYCEAE } \\
\hline Dinoflagelado desnudo N. I. & $\mathrm{X}$ & $\mathrm{X}$ \\
\hline Gymnodinium sp. & $\mathrm{X}$ & $\mathrm{x}$ \\
\hline Peridinium sp. & $\mathrm{X}$ & $\mathrm{X}$ \\
\hline
\end{tabular}

Es de suponer que el aporte de nutrientes por las lluvias, desde la atmósfera y/o por arrastre desde los terrenos circundantes (con áreas cultivadas) fue importante para el desarrollo del fitoplancton. La limitación del fósforo fue marcada, a diferencia del nitrógeno (en sus distintas formas) que en algunos casos fue limitante. En tal ocasión, durante el período en que predominaron las cianofíceas filamentosas heterocistadas se observó un aumento en la frecuencia de filamentos con heterocistes, en coincidencia con un período de notoria sequía. 
Agradecimientos - Al director del Centro de Ecologia Aplicada del litoral, Prof. Juan J. Neiff por el apoyo brindado en la realización del trabajo, a los señores Luis A. Benetti y Miguel A. Solari la valiosa colaboración en las tareas de campo, a Abel O. Ramos y Julio R. Cáceres la realización de los análisis de nutrientes, a Francisco G. Benítez el entintado de las figuras y al personal del Centro Tecnológico de Producción (Convenio Gobierno provincia de Corrientes-Gobierno de Japón) el habernos proporcionado los datos meteorológicos.

\section{REFERENCIAS BIBLIOGRAFICAS}

APHA, 1985, Standard methods for the examination of water and wastewater. APHA, ANWA and WPCF, Washington, $874 \mathrm{p}$.

BARBIERO, R. P., 1993, A contribution to the life history of the planktonic cyanophyte, Gloeotrichia echinulata. Arch. Hydrobiol., 127(1): 87-100.

BARBIERO, R. P. \& WELCH, E. B., 1992. Contribution of benthic blue-green algal recruitment to lake populations and phosphorus translocation. Freshwater Biology, 27: 249-260.

BARBIERO, R. P. \& KANN, J., 1994, The importance of benthic recruitment to the population development of Aphanizomenon flos-aqune and internal loading in a shallow lake. Journal of Planiton Research, 16(11): 1581-1588.

BLOMQVIST, P., PETTERSSON, A. \& HYENSTRAND, P., 1994, Ammonium-nitrogen: A key regulatory factor causing dominance of non-nitrogen-fixing cyanobacteria in aquatic systems. Arch. Hydrobiol., 132(2): 141-164.

BONETTO, A. A., CORRALES, M. A., VARELA, M. E., RIVERO, M. M., BONETTO, C. A \& y ZALOCAR, Y., 1978a, Estudios limnológicos en la cuenca del Riachuelo. II. Lagunas Totoras y González. ECOSUR, 5(9): 17-55.

BONETTO, A. A., NEIFF, J. J., POI de NEIFF, A., VARELA, M. E., CORRALES, M. A. \& ZALOCAR, Y., 1978b, Estudios limnológicos en la cuenca del Riachuelo (Corrientes, Argentina) III: Laguna "La Brava". ECOSUR, 5(9): 57-84.

BRUNIARD, E. D.,1981, El clima de las planicies del norte argentino. Facultad de Humanidades de la U.N.N.E. Resistencia, Chaco, Vol. I-II, 379p. (Tesis doctoral).

CARO, P. M., BONETTO, C. A. \& ZALOCAR, Y., 1979 , Producción primaria del fitoplancton de Lagunas del Noroeste de la provincia de Corrientes. ECOSUR, 6(11): 83-100.

ESTEVES, F. A., 1988, Fundamentos de Limnologia. Ed. Interciência, Rio de Janeiro, 575p.

GARCIA de EMILIANI, M. O., 1980, Fitoplancton de una laguna del valle aluvial del Paraná medio ("Los Matadores", Santa Fé, Argentina). I. Estructura y distribución en relación a factores ambientales. Ecología, 4: $127-140$

GARCIA de EMILIANI, M. O., 1990, Variaciones diurnas del fitoplancton (laguna "El tigre", llanura aluvial del Paraná medio, Argentina). Rev. Asoc. Cienc. Nat. Litoral, 21: 49-60.
GARCIA de EMILIANI, M. O., 1993, Seasonal succession of phytoplankton in a lake of he Paraná river floodplain, Argentina. Hydrobiologia, 264: 101-114.

GIANI, A. \& LEONARDO, I. M., 1988, Distribuição vertical de algas fitoplanctônicas no reservatório da Pampulha (Belo Horizonte, MG). Acta Limnol. Brasil., 11: 387-404.

HUSZAR, V. L. M., 1989. Considerações sobre o fitoplâncton da lagoa de Juturnaíba, Araruama, Estado do Rio de Janeiro, Brasil. Rev. Brasil. Biol., 49(1): 107-123.

HUTCHINSON, G. E., 1957, A Treatise on Limnology. Geography, physics and chemistry. J. Wiley \& Sons, Inc., New York, Vol. 1, 1015p.

HUTCHINSON, G. E., 1967, A Treatise on Limnology. Introduction to lake biology and the limnoplankton. J. Wiley \& Sons, Inc., New York, Vol. 2, 1015p.

LEWIS, W. M., 1978a, Dynamics and succession of the phytoplankton in a tropical lake: Lake Lanao, Philippinas. J. Ecol., 66: 849-880.

LEWIS, W. M.,1978b, Analysis of succession in a tropical phytoplankton community and new measure of succession rate. Amer. Natur., 112(984): 401-414.

LUND, J. W. G., KIPLING, C. \& LE CREN, E. D., 1958, The inverted microscope method of estimating algal numbers and statiscal basis of estimating by counting. Hydrobiologia, 11:143-170.

MARINHO, M. M. \& HUSAR, V. L. M., 1990, Estrutura da comunidade fitoplanctônica da lagoa de Juturnaíba, Araruama, RJ, Brasil: Uma comparação entre o centro da região limnética, tributários e canal de drenagem. Rev. Brasil. Biol., 50(2): 313-325.

MARINHO, M. M., HUSZAR, V. L. M. \& SAMPAIOPINTO, G. F., 1993. Estrutura da comunidade fitoplanctônica da lagoa de Juturnaíba, Araruama, RJ, Brasil: Uma comparação entre os períodos anterior e posterior à construção da Barragem no rio São João. Rev. Brasil. Biol., 53(3): 453-467.

MATVEEV, V., MARTINEZ, C. C., FRUTOS, S. M. \& ZALOCAR de DOMITROVIC, Y., 1992, Population control in planktonic crustaceans of a subtropical lake during seasonal succession. Arch. Hydrobiol., 124(1): 1-18.

OLEKSOWICZ, A. S., 1987, Does there exist competition between phytoplankton and littoral plant vegetation in lake ecosystem? Arch. Hydrobiol. Suppl., 73(4): 575-585.

PRESTON, T., STEWART, W. D. P. \& REYNOLDS, C. S., 1980, Bloom-forming cyanobacterium Microcystis aeruginosa over-winters on sediment surface. Nature, 288: 365-367.

REYNOLDS, C. S., 1972, Growt, gas vacuolation and bouyancy in a natural population of planktonic bluegreen alga. Freshwater Biology, 2: 87-106.

REYNOLDS, C. S., 1984, The ecology off reshwater phytoplankton. Cambridge University Press, Cambridge, $384 \mathrm{p}$.

REYNOLDS, C. S., 1988, Functional morphology and the adaptive strategies of freshwater phytoplankton. In: C. D. Sandgren (ed.). Growth and reproductive strategies of freshwater phytoplankton, Cambridge University Press, Cambridge, 388-433. 
ROTT, E., 1981, Some results from phytoplankton counting intercalibrations. Schweitz Z. Hydrol., 43: 34-61.

SCHMIDT, G. W., 1970, Numbers of bacterias and algae and their interrelations in some Amazonian waters. Amazoniana, 2(4): 393-400.

SENICHKIN, L. G., 1978, A procedure for calculating the volumes of algal cells in Plankton. Hydrobiological Journal., 14(5): 87-90.

SHANNON, C. \& WEAVER, W., 1963, The mathematical theory of communication. Univ. Illinois Press. Urbana, $117 \mathrm{p}$.

SIRENKO, L. A., CHERNOUSOVA, V. M, ARENDARCHUK, V. V. \& KOZITSKAYA, V. N., 1969, Factors of mass development of blue-green algae. Hydrobiological Journal, 5(3): 1-10.

SOKAL, R. \& ROHLF, F. J., 1979, Biometría. Principios y métodos estadísticos en la investigación biológica. Ed. Blume, Madrid, 832p.

TALLING, J. F., 1986, The seasonality of phytoplankton in African lakes. Hydrobiologia, 138: 139-160.
UTERMÖHL, H., 1958, Zur Vervollkomrnnung ver quantitativen Phytoplankton-Methodic. Mitt. Int. Verein. Limnol., 9: 1-38.

WETZEL, R. G., 1981, Limnología. Ed. Omega, Barcelona, $679 \mathrm{p}$.

ZALOCAR de DOMITROVIC, Y., 1990, Efecto de las fluctuaciones del nivel hidrométrico sobre el fitoplancton en tres lagunas isleñas en al área de confluencia de los ríos Paraná y Paraguay. ECOSUR, 16(27): 1-23.

ZALOCAR de DOMITROVIC, Y., 1992, Fitoplancton de ambientes inundables del río Paraná (Argentina). Rev. Hydrobiol. Trop., 25(3): 177-188.

ZALOCAR de DOMITROVIC, Y., 1993, Fitoplancton de una laguna vegetada por Eichhornia crassipes en el valle de inundación del río Paraná (Argentina). Ambiente Subtropical, 3: 39-67.

ZALOCAR de DOMITROVIC, Y., VALLEJOS, E. R. \& PIZARRO, H. N., 1986, Aspectos ecológicos de la ficoflora de ambientes acuáticos del Chaco Oriental (Argentina). Ambiente Subtropical, 1: 92-111. 